\title{
Continuous Phase Modulation of F-QPSK-B Signals
}

\author{
Hyung Chul Park, Member, IEEE, Kwyro Lee, Senior Member, IEEE, and Kamilo Feher, Fellow, IEEE
}

\begin{abstract}
A continuous phase modulation (CPM) implementation alternative of a recently standardized class of Feher-patented quadrature phase-shift keying (F-QPSK-B) modulation is proposed. Based on the fact that the F-QPSK-B signal has a quasiconstant envelope and continuous phase characteristics, it is shown that it can indeed be generated by the CPM scheme. For example, an F-QPSK-B signal can be fully generated using an existing FM-based transmitter with a modulation index of 0.5 . Furthermore, a premodulation filter and an alternating change monitor differential encoder for the continuous-phase-modulated F-QPSK-B signal to be fully compatible with the I/Q modulated F-QPSK-B signal are proposed, allowing direct symbol-bysymbol coherent detection without the use of any special decoding schemes inherent in all CPM schemes. It is shown that the power spectral density and eye diagram of the continuous-phasemodulated F-QPSK-B signal are practically the same as those of the I/Q modulated F-QPSK-B signal. By utilizing CPM characteristics, an optimum maximum-likelihood (ML) coherent receiver for the F-QPSK-B signal is proposed. It is shown that the biterror-rate performances of the optimum ML coherent detection, symbol-by-symbol coherent detection, and noncoherent detection of the continuous-phase-modulated F-QPSK-B signal are almost the same as those of the I/Q modulated F-QPSK-B signal.
\end{abstract}

Index Terms-Continuous phase modulation (CPM), Feherpatented quadrature phase-shift keying (F-QPSK), maximumlikelihood (ML) detection, premodulation filter.

\section{INTRODUCTION}

D URING the last few years, there have been extensive research efforts for simple, low-cost, and low-power wireless transceivers with specific emphasis on the implementation of a single-chip transceiver [1]-[4]. For the efficient implementation of single-chip transceivers, continuous phase modulation (CPM) using the FM scheme is used to reduce the cost and complexity of transmitters with an I/Q modulation/up-conversion scheme [1], [2]. The I/Q modulation scheme has some disadvantages. First, it requires various signal mixing. This means that I/Q modulation architecture is complex and requires excessive power consumption. Second, it is difficult to match the $I$ and $Q$ signals because of gain and phase mismatches in the local oscillators, low-pass filters (LPFs), and mixers [5].

Manuscript received May 1, 2003; revised March 1, 2004, May 27, 2004, January 13, 2005, October 23, 2005, and November 3, 2005. This work was supported by the Micro Information and Communication Remote Objectoriented System (MICROS) Research Center. The review of this paper was coordinated by Prof. G. Saulnier.

H. C. Park is with the Department of Information, Communication, and Computer Engineering, Hanbat National University, Daejeon 305-719, Korea (e-mail: chori@dimple.kaist.ac.kr).

K. Lee is with the MICROS Research Center, Department of Electrical Engineering and Computer Science, Korea Advanced Institute of Science and Technology, Daejeon 305-701, Korea (e-mail: krlee@ee.kaist.ac.kr).

K. Feher is with the Digcom, Inc., El Macero, CA 95618 USA (e-mail: feherk@yahoo.com).

Digital Object Identifier 10.1109/TVT.2006.883766
The CPM scheme does not require I/Q signals; therefore, transmitters using the scheme are less complex, less costly, and use less power. Furthermore, an additional power amplifier is not necessary when the carrier produced by the RF local oscillator is directly modulated at the desired transmit power level [6]. For these reasons, the CPM scheme is used to implement many lowcomplexity, low-cost, and low-power consumption transmitters, such as Bluetooth [2], [7] and DECT [8], [9].

There has also been an increasing need for both power and spectrum efficient modulation techniques for telemetry systems. Multiyear studies by the U.S. Department of Defense, NASA, AIAA, and the International Consultative Committee for Space Data Systems (CCSDS) have confirmed that Feherpatented quadrature phase-shift keying (F-QPSK) technologies [10]-[16] offer the most spectrally efficient and robust (smallest degradation from ideal theory) bit-error-rate (BER) performance of nonlinear amplification (NLA, saturation mode operation of high-power amplifier)-RF power efficient systems. Based on numerous F-QPSK airplane-to-ground, ground-toground, and satellite tests in the 1 to $600 \mathrm{Mb} / \mathrm{s}$ range, F-QPSK has been specified in the new telemetry standard known as IRIG 106-00 [17] and has been recommended by the CCSDS for use in high-speed space communication systems. Since the F-QPSK-B modulation was proposed, only the I/Q modulation scheme has been used for transmitter implementation.

This paper presents the CPM method for the F-QPSK-B signal. Based on the observation that the F-QPSK-B signal has quasi-constant envelope and continuous-phase- characteristics, we first show that the F-QPSK-B can be interpreted as a kind of CPM. We then propose a CPM scheme for the F-QPSK-B signal using FM. This is especially important because the F-QPSK-B signal can be easily generated by systems already in service that do not have the capability for I/Q modulation.

In Section II, the F-QPSK-B modulation is reviewed. In Section III, the F-QPSK-B signal is interpreted as a CPM. Both the modulation index and premodulation filter are specified for the CPM representation of the F-QPSK-B signal. The alternating change monitor differential encoder (ACMDE) is also proposed. CPM of the F-QPSK-B signal requires an ACMDE to achieve the same phase behavior as that produced by the I/Q modulated F-QPSK-B signal. In Section IV, power spectral density (PSD), eye diagram, and BER performances of the continuous-phase-modulated F-QPSK-B signal are evaluated and compared with those of the conventional I/Q modulated F-QPSK-B signals. For the BER performance comparison, optimum maximum-likelihood (ML) coherent detection, symbol-by-symbol coherent detection, and noncoherent detection schemes are used. Note that the optimum ML coherent detection is proposed utilizing the CPM characteristics of the F-QPSK-B signal. Finally, conclusions are presented in Section V. 


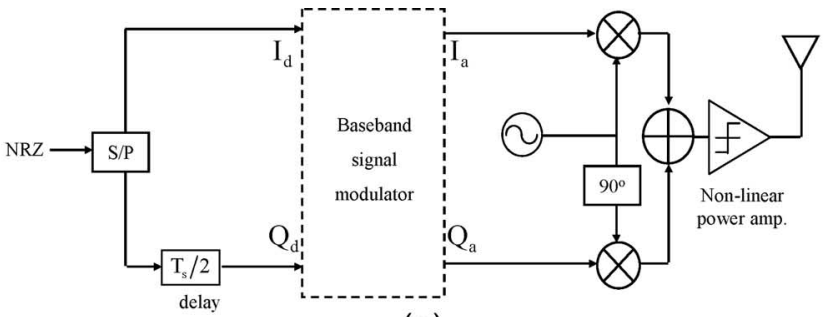

(a)

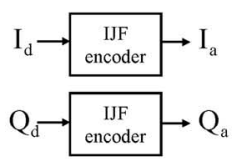

(b)

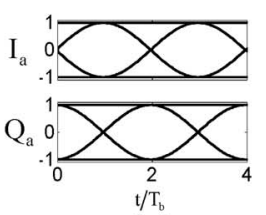

(c)

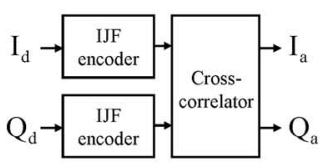

(e)

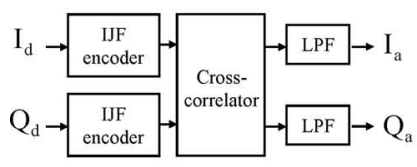

(h)

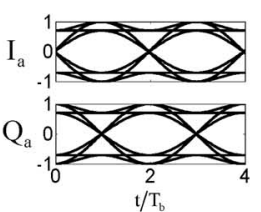

(f)

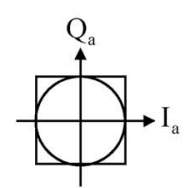

(d)

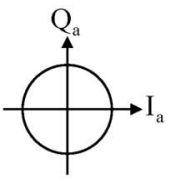

(g)

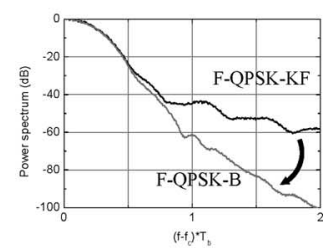

(i)
Fig. 1. F-QPSK modulation techniques. (a) Block diagram of F-QPSK-1, F-QPSK-KF, and F-QPSK-B modulations. (b) Baseband signal modulator for F-QPSK-1. (c) Eye diagram of F-QPSK-1 signal. (d) Signal constellation of F-QPSK-1 signal. (e) Baseband signal modulator for F-QPSK-KF. (f) Eye diagram of F-QPSK-KF signal. (g) Signal constellation of F-QPSK-KF signal. (h) Baseband signal modulator for F-QPSK-B. (i) PSD of F-QPSK-KF and F-QPSK-B signal.

\section{F-QPSK-B MODULATION}

As shown in Fig. 1(a) and (h), the F-QPSK-B modulation is a kind of intersymbol interference (ISI) and jitter-free OQPSK modulation scheme, which is composed of a cross correlator and an LPF [10]-[15].

In F-QPSK-B modulation, the signal modulated with ISI and jitter-free (IJF) signal encoder, which is designated as F-QPSK-1 [Fig. 1(b)], is written as

$$
\begin{array}{r}
s_{\mathrm{F}-\mathrm{QPSK}-1}(t)=I_{\mathrm{F}-\mathrm{QPSK}-1}(t) \cos \left(\omega_{c} t\right)+Q_{\mathrm{F}-\mathrm{QPSK}-1}(t) \sin \left(\omega_{c} t\right) \\
n T_{s}<t<(n+1 / 2) T_{s}
\end{array}
$$

where $x(k)$ and $y(k)$ denote the binary input data, \pm 1 , and $T_{s}\left(=2 T_{\mathrm{b}}\right)$ denotes the symbol period. Fig. 1(c) shows the eye diagram of the F-QPSK-1 signals. The signal modulated with an IJF signal encoder has a 3-dB envelope fluctuation, as shown in Fig. 1(d). This causes a spectrum regrowth after the signal passes through the nonlinear amplifier. However, the cross correlator in the F-QPSK-B modulation reduces the 3-dB envelope fluctuation to near $0 \mathrm{~dB}$ with an amplitude parameter $A=1 / \sqrt{2}$ [13]. ${ }^{1}$ This process, designated as F-QPSK-KF [Fig. 1(e)], allows us to use a nonlinear amplifier, such as a class-C amplifier, without much spectrum regrowth, leading to higher power efficiency [15]. The eye diagram and signal constellation of the F-QPSK-KF signals are shown in Fig. 1(f) and $(\mathrm{g})$.

Spectrum efficiency is further improved by using an LPF on the F-QPSK-KF signal, which is designated as F-QPSK-B [Fig. 1(h)]. The spectral sidelobes of the F-QPSK-B signal are significantly lower than those of the F-QPSK-KF signal due to the low-pass-filtering sidelobes, which is shown in Fig. 1(i). It is interesting to note that analog low pass filtering, using a Butterworth filter, does not change the ISI/IJF and quasiconstant envelope characteristics of the F-QPSK-KF signal.

\section{CPM OF THE F-QPSK-B SIGNAL}

\section{A. CPM Interpretation of the F-QPSK-B Signal}

In general, CPM has the following characteristics. First, the CPM signal has a constant envelope. Second, the information is stored in the continuously changing phase. Since the F-QPSK-B signal has a quasi-constant envelope, its phasor moves along the unit circle in the signal constellation [13], [15]. Since the $I$ and $Q$ signals in the F-QPSK-B modulation must be highly correlated to obtain a quasi-constant envelope, let us start to analyze the correlation with three consecutive binary data bits, i.e., two consecutive $Q$ data bits with $I$ data bit in between, and vice versa.

1) F-QPSK-B Modulation With Three Consecutive Binary Data Bits: Let us show that three consecutive binary data bits determine a phase point uniquely at the middle of the symbol period. Fig. 2 lists all possible phase points at $t=2 n T_{\mathrm{b}}$ for three consecutive binary data bits $\left(Q_{n-1}, I_{n}, Q_{n}\right)$. The possible phase points are generated according to the waveform shaping rule for the F-QPSK-B signal. When two consecutive data bits for the $Q$ channel do not change, either $1 / \sqrt{2}$ or $-1 / \sqrt{2}$ is assigned to their modulated signal at the middle of the symbol period $\left(t=2 n T_{\mathrm{b}}\right)$, depending on whether they are one or zero. In addition, $1 / \sqrt{2}$ or $-1 / \sqrt{2}$ is assigned to the modulated

where

$$
\begin{aligned}
I_{\mathrm{F}-\mathrm{QPSK}-1}(t) & =\sum_{k=0}^{n+1} x(k) \cdot p\left(t-k \cdot T_{s}\right) \\
Q_{\mathrm{F}-\mathrm{QPSK}-1}(t) & =\sum_{k=0}^{n} y(k) \cdot p\left(t-k \cdot T_{s}-T_{s} / 2\right) \\
p(t) & = \begin{cases}\frac{1}{2}\left(1+\cos \left(\frac{\pi t}{T_{s}}\right)\right), & |t| \leq T_{s} \\
0, & \text { elsewhere }\end{cases}
\end{aligned}
$$

\footnotetext{
${ }^{1}$ In F-QPSK-KF and F-QPSK-B modulation, the envelope of the modulated signal is determined by the baseband waveforms. These baseband waveforms are dependent on the amplitude parameter $A$, which are shown in Table II and [13]. For example, when the modulated signal's phase changes from 0 to $+\pi / 4$ during the $T_{b}$ interval, the $I$ channel waveform is equal to $1-(1-A) \sin ^{2}\left(\pi t /\left(2 T_{b}\right)\right)$, and the $Q$ channel waveform is equal to $A$. $\sin \left(\pi t /\left(2 T_{b}\right)\right)$. We find that when $A$ is equal to $1 / \sqrt{2}$, the modulated signal has a quasi-constant envelope (for details, see [13]).
} 

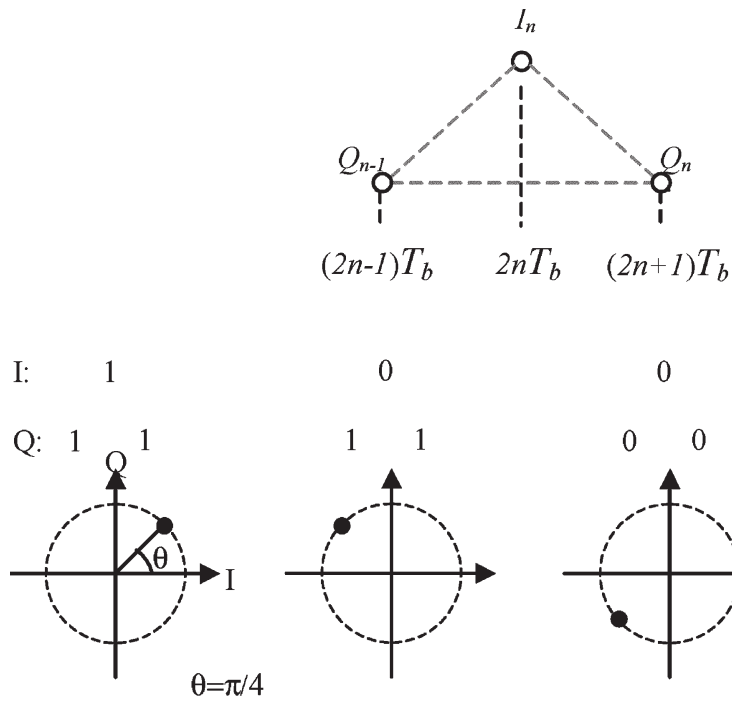

0

0

1
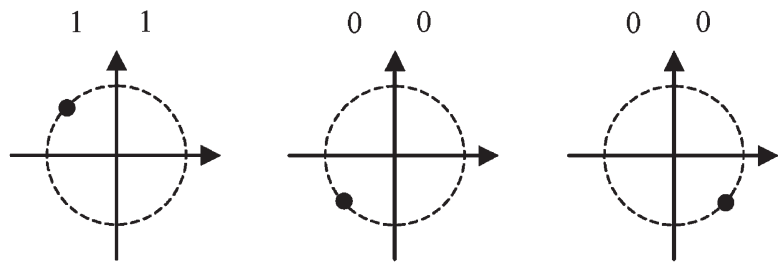

I: $\quad 1$

0

0

1
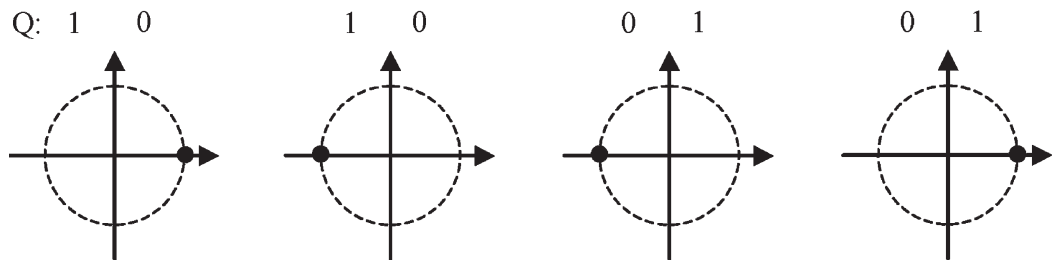

Fig. 2. Allowed phase points (solid dots) of F-QPSK-B signal at $t=2 n T_{\mathrm{b}}$ for three consecutive binary data bits $\left(Q_{n-1}, I_{n}, Q_{n}\right)$.

signal in the $I$ channel depending on its data. This generates a modulated signal envelope of one, given by $\sqrt{I^{2}(t)+Q^{2}(t)}$. When two consecutive data bits for the $Q$ channel change from one to zero, or vice versa, zero is assigned to the modulated signal in the $Q$ channel at the middle of the symbol period $\left(t=2 n T_{\mathrm{b}}\right)$ and, depending on its data, 1 or -1 is assigned to the modulated signal in the $I$ channel, again making the envelope of the modulated signal equal to one. Data bits $\left(I_{n}, Q_{n}, I_{n+1}\right)$ allow a similar set of phase points at $t=(2 n+$ 1) $T_{\mathrm{b}}$. The only difference is that $\left(I_{n}, Q_{n}, I_{n+1}\right)$ allow $+\pi / 2$, $-\pi / 2$, whereas $\left(Q_{n-1}, I_{n}, Q_{n}\right)$ allow zero, $\pi$. Therefore, three consecutive binary data bits determine the eight phase points allowed at $t=n T_{\mathrm{b}}$, which is given by $\phi\left(n T_{\mathrm{b}}\right)=k \cdot(\pi / 4)$, $(k=0, \ldots, 7)$.

2) F-QPSK-B Modulation With Four Consecutive Binary Data Bits: Now, let us look at four consecutive binary data bits as shown in Fig. 3. In F-QPSK-B modulation, four consecutive binary data bits $\left(Q_{n-1}, I_{n}, Q_{n}, I_{n+1}\right)$ determine two phase points at $t=2 n T_{\mathrm{b}}$ and $t=(2 n+1) T_{\mathrm{b}}$, respectively, and a single-phase transition path between them. Single-phase transition is written as

$$
\Delta \phi_{2 n}=\phi\left((2 n+1) T_{\mathrm{b}}\right)-\phi\left(2 n T_{\mathrm{b}}\right), \quad n=0,1,2, \ldots
$$

The F-QPSK-B signal can only have five single-phase transition values, which are given as $\pm \pi / 2, \pm \pi / 4$, and 0 . We can find that the phase of the F-QPSK-B signal changes continuously from $\phi\left(2 n T_{\mathrm{b}}\right)$ to $\phi\left((2 n+1) T_{\mathrm{b}}\right)$.

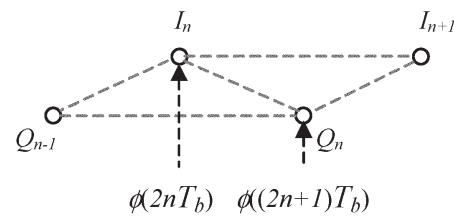

(a)
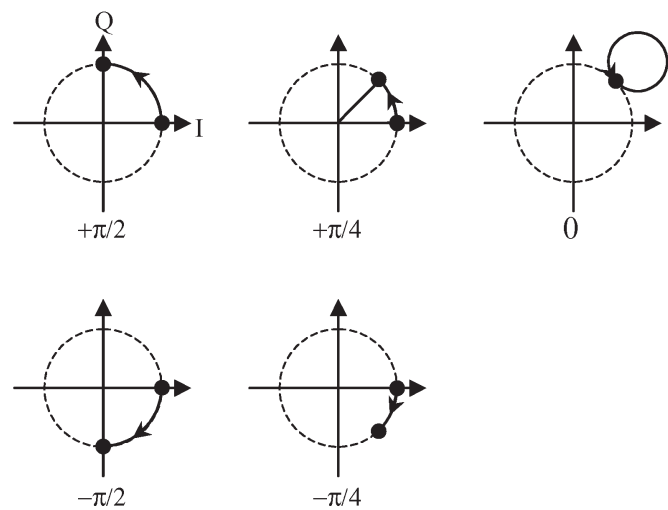

(b)

Fig. 3. Single-phase transition diagrams between $2 n T_{\mathrm{b}} \leq t \leq(2 n+1) T_{\mathrm{b}}$ for four consecutive binary data bits $\left(Q_{n-1}, I_{n}, Q_{n}, I_{n+1}\right)$. Note that only five single-phase transitions are allowed, i.e., $\pm \pi / 2, \pm \pi / 4$, and 0 .

3) F-QPSK-B Modulation With Five Consecutive Binary Data Bits: Extending the interpretation presented above, we see that five consecutive binary data bits $\left(Q_{n-1}, I_{n}, Q_{n}\right.$, $\left.I_{n+1}, Q_{n+1}\right)$ determine three phase points at the symbol time, $t=2 n T_{\mathrm{b}}, \quad(2 n+1) T_{\mathrm{b}}, \quad$ and $2(n+1) T_{\mathrm{b}}, \quad$ and two-phase 

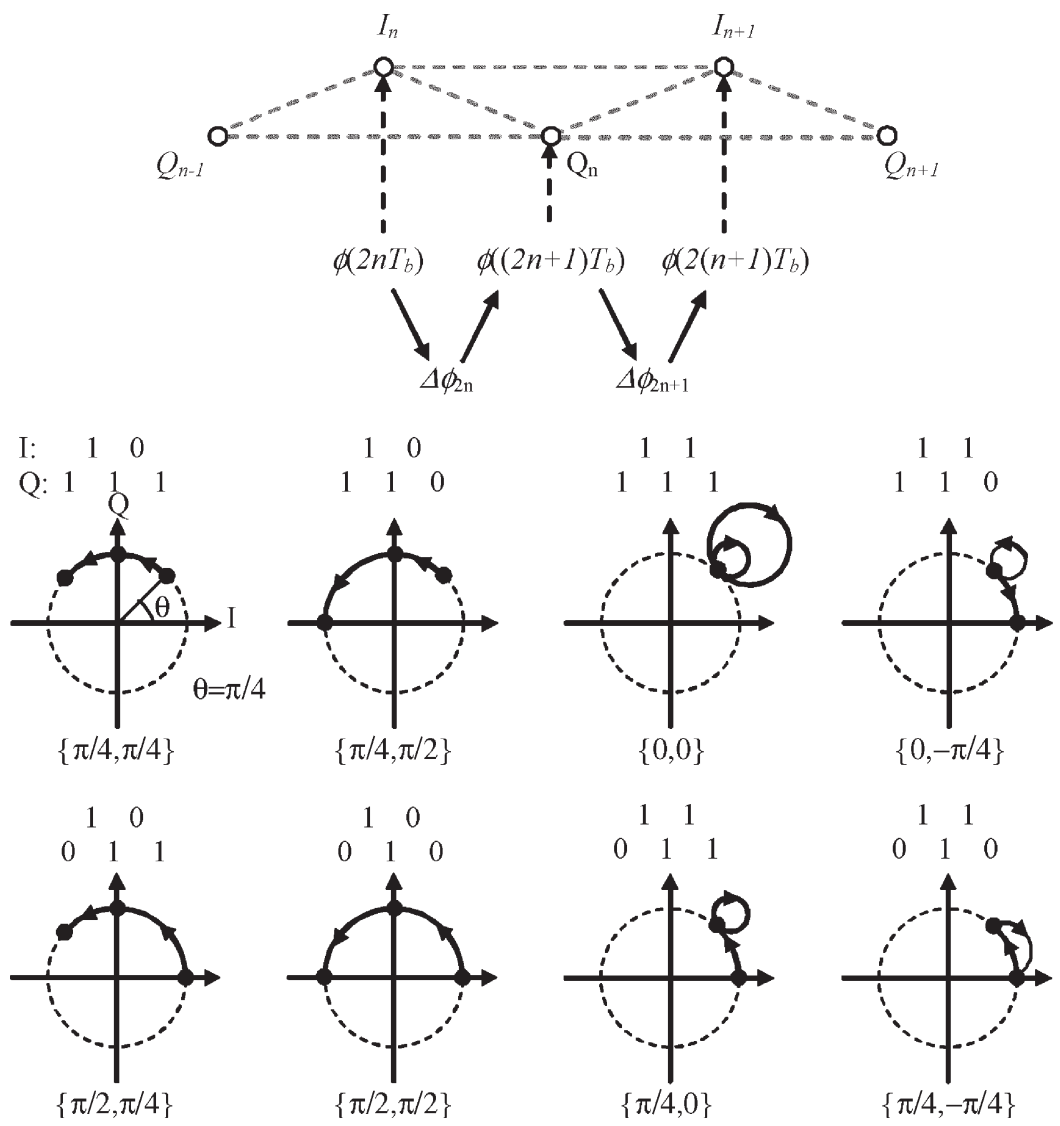

Fig. 4. Two-phase transition diagram examples between $2 n T_{\mathrm{b}} \leq t \leq 2(n+1) T_{\mathrm{b}}$ for five consecutive binary data bits $\left(Q_{n-1}, I_{n}, Q_{n}, I_{n+1}, Q_{n+1}\right)$.

TABLE I

Allowed Two-Phase Transitions

\begin{tabular}{ccc||ccc}
\hline \hline & $\Delta \phi_{2 \mathrm{n}}$ & $\Delta \phi_{2 \mathrm{n}+1}$ & & $\Delta \phi_{2 \mathrm{n}}$ & $\Delta \phi_{2 \mathrm{n}+1}$ \\
\hline 1 & $-\pi / 2$ & $-\pi / 2$ & 9 & 0 & $+\pi / 4$ \\
2 & $-\pi / 2$ & $-\pi / 4$ & 10 & $+\pi / 4$ & $-\pi / 4$ \\
3 & $-\pi / 4$ & $-\pi / 2$ & 11 & $+\pi / 4$ & 0 \\
4 & $-\pi / 4$ & $-\pi / 4$ & 12 & $+\pi / 4$ & $+\pi / 4$ \\
5 & $-\pi / 4$ & 0 & 13 & $+\pi / 4$ & $+\pi / 2$ \\
6 & $-\pi / 4$ & $+\pi / 4$ & 14 & $+\pi / 2$ & $+\pi / 4$ \\
7 & 0 & $-\pi / 4$ & 15 & $+\pi / 2$ & $+\pi / 2$ \\
8 & 0 & 0 & & & \\
\hline \hline
\end{tabular}

transitions. As shown in Fig. 4, the two-phase transitions are composed of two consecutive single-phase transitions: $\Delta \phi_{2 n}$ and $\Delta \phi_{2 n+1}$. Fig. 4 also shows some of the allowed twophase transitions; all allowed two-phase transitions are shown in Table I. Random combination of the five allowed singlephase transitions seemingly produces 25 possible two-phase transitions. It is very important to note that a much lower number of allowed two-phase transitions, i.e., 15, actually exist. The discrepancy in the number of allowed two-phase transitions results from the fact that the F-QPSK-B modulation has memory [18]. The aforementioned interpretations of the F-QPSK-B signal lead us to interpret F-QPSK-B as a kind of CPM, allowing the possibility of generating an F-QPSK-B signal using an FM scheme.

\section{B. Modulation Index for CPM of F-QPSK-B}

In general, the CPM signal, now including the F-QPSK-B signal, $s(t)$ is represented as [19]

$$
\begin{aligned}
s(t) & =\sqrt{2 E / T_{\mathrm{b}}} \cdot \cos \left(2 \pi f_{c} t+\phi(t)+\phi_{0}\right) \\
n T_{\mathrm{b}} \leq t \leq(n+1) T_{\mathrm{b}} & \\
\phi(t) & =2 \pi h \sum_{k=-\infty}^{n} a_{k} q\left(t-k T_{\mathrm{b}}\right) \\
& =2 \pi h \sum_{k=-\infty}^{n}\left[a_{k} \int_{0}^{t-k T_{\mathrm{b}}} g(\tau) d \tau\right] \\
g(t) & =0, \quad t\langle 0, t\rangle L T_{\mathrm{b}}
\end{aligned}
$$

where $h$ denotes the modulation index, $g(t)$ is the impulse response of the premodulation filter, $a_{k}$ is the input data, and $\phi_{0}$ is a constant.

The single-phase transition of the F-QPSK-B signal, described in (2), is then written as

$$
\begin{aligned}
\Delta \phi_{\mathrm{n}} & =\phi\left((n+1) T_{\mathrm{b}}\right)-\phi\left(n T_{\mathrm{b}}\right) \\
& =2 \pi h \sum_{k=-\infty}^{n} a_{k}\left[q\left((n+1-k) T_{\mathrm{b}}\right)-q\left((n-k) T_{\mathrm{b}}\right)\right] \\
& =2 \pi h \sum_{k=-\infty}^{n} a_{k} \int_{(n-k) T_{\mathrm{b}}}^{(n+1-k) T_{\mathrm{b}}} g(\tau) d \tau \\
& =2 \pi h Q(t)
\end{aligned}
$$


If all elements in binary data sequence $\left\{a_{k}\right\}$ in (4) are the same, i.e., $\{\ldots, 1,1, \ldots, 1, \ldots\}$ or $\{\ldots,-1,-1, \ldots,-1, \ldots\}$, then $\Delta \phi_{\mathrm{n}}$ attains the maximum magnitude value corresponding to the maximum single-phase transition of the F-QPSK-B signal, i.e., $\pi / 2$. In this case, $Q(t)$ in (4) is identical to the entire integration of $g(t)$ [20]. Hence, $Q(t)$ becomes equal to $1 / 2$. This analysis shows that modulation index $h$ for the CPM of F-QPSK-B is equal to 0.5 .

\section{Premodulation Filter for CPM of F-QPSK-B}

The premodulation filter $g(t)$ can be derived from the instantaneous frequency of the F-QPSK-B signal, i.e., the derivative of the F-QPSK-B signal's phase. However, there are difficulties to derive $g(t)$ from the instantaneous frequency of the F-QPSK-B signal. First, F-QPSK-B modulation uses an analog LPF in the $I$ and $Q$ signal paths. The infinite impulse response characteristic of the analog LPF makes it difficult to derive $g(t)$ from the instantaneous frequency of the F-QPSK-B signal. The premodulation filter for the CPM of the F-QPSK-B signal may be found by utilizing the premodulation filter for the CPM of the F-QPSK-KF signal. However, there exists a discontinuity in the first derivative of the F-QPSK-KF signal [13]. For example, when the input data sequence $\left(I_{n-1}, Q_{n-1}, I_{n}, Q_{n}, I_{n+1}\right)$ is equal to $(0,0,1,1,1)$, the corresponding F-QPSK-KF signal is written as

$I$ channel:

$$
\begin{cases}\sin \left(\frac{\pi}{2 T_{\mathrm{b}}}\left(t-(2 n-1) T_{\mathrm{b}}\right)\right), & (2 n-1) T_{\mathrm{b}}<t<2 n T_{\mathrm{b}} \\ 1-\left(1-\frac{1}{\sqrt{2}}\right) & \\ \times \sin ^{2}\left(\frac{\pi}{2 T_{\mathrm{b}}}\left(t-2 n T_{\mathrm{b}}\right)\right), & 2 n T_{\mathrm{b}}<t<(2 n+1) T_{\mathrm{b}}\end{cases}
$$

$Q$ channel:

$$
\begin{cases}-\cos \left(\frac{\pi}{2 T_{\mathrm{b}}}\left(t-(2 n-1) T_{\mathrm{b}}\right)\right), & (2 n-1) T_{\mathrm{b}}<t<2 n T_{\mathrm{b}} \\ \frac{1}{\sqrt{2}} \sin \left(\frac{\pi}{2 T_{\mathrm{b}}}\left(t-2 n T_{\mathrm{b}}\right)\right), & 2 n T_{\mathrm{b}}<t<(2 n+1) T_{\mathrm{b}} .\end{cases}
$$

In (5), we find that the $Q$ channel signal is not differentiable at $t=2 n T_{\mathrm{b}}$, which is shown in Fig. 5. This makes it hard to derive an exact premodulation filter for the CPM of the F-QPSK-KF signal. In order to overcome this difficulty, we propose the F-QPSK- $\mathrm{E}^{2}$ modulation. It modifies four out of seven basic waveforms of the F-QPSK-KF modulation, which are presented in Table II. F-QPSK- ${ }^{2}$ modulation has the following advantages over F-QPSK-KF modulation. First, F-QPSK-E ${ }^{2}$ modulation guarantees continuity in the first derivative of the modulated signal, which is shown in Fig. 5, while the power spectrum of the F-QPSK-E ${ }^{2}$ signal is almost the same as that of the F-QPSK-KF signal. Second, the BER performance of the F-QPSK-E ${ }^{2}$ is better than that of the F-QPSK-KF. For example, the F-QPSK-E ${ }^{2}$ achieves an improvement of about $0.5 \mathrm{~dB}$ at a BER of $10^{-4}$ over that of the F-QPSK-KF.

In order to calculate the premodulation filter for CPM of the F-QPSK-E ${ }^{2} g_{0}(t)$, it is necessary to consider the fol-

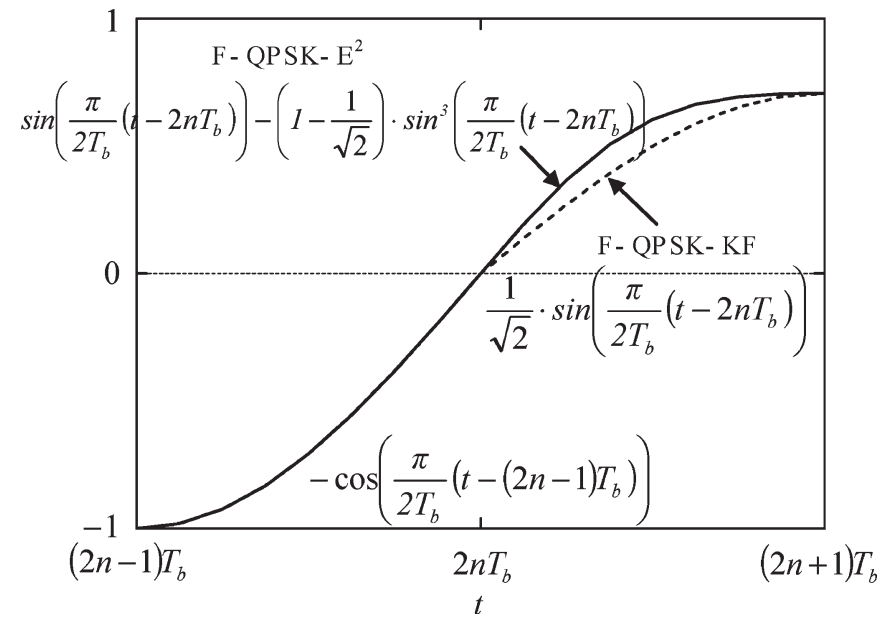

Fig. 5. I/Q channel waveforms of F-QPSK-KF and F-QPSK-E ${ }^{2}$ signal $(A=1 / \sqrt{2})$.

TABLE II

COMPARISON OF F-QPSK-KF AND F-QPSK-E ${ }^{2}$ WAVEFORMS $\left(0<t<T_{\mathrm{b}}\right)$

\begin{tabular}{rr}
\hline \hline F-QPSK-KF & F-QPSK-E ${ }^{2}$ \\
\hline $1-(1-A) \cdot \cos ^{2}\left(\frac{\pi}{2 T_{b}} t\right)$ & $1-\frac{1}{2} \cdot \cos ^{2}\left(\frac{\pi}{2 T_{b}} t\right)+\left(A-\frac{1}{2}\right) \cdot \cos ^{4}\left(\frac{\pi}{2 T_{b}} t\right)$ \\
$A \cdot \sin \left(\frac{\pi}{2 T_{b}} t\right)$ & $\sin \left(\frac{\pi}{2 T_{b}} t\right)-(1-A) \cdot \sin ^{3}\left(\frac{\pi}{2 T_{b}} t\right)$ \\
$A \cdot \cos \left(\frac{\pi}{2 T_{b}} t\right)$ & $\cos \left(\frac{\pi}{2 T_{b}} t\right)-(1-A) \cdot \cos ^{3}\left(\frac{\pi}{2 T_{b}} t\right)$ \\
$1-(1-A) \cdot \sin ^{2}\left(\frac{\pi}{2 T_{b}} t\right)$ & $\sin ^{2}\left(\frac{\pi}{2 T_{b}} t\right)+\left(A-\frac{1}{2}\right) \cdot \sin ^{4}\left(\frac{\pi}{2 T_{b}} t\right)$ \\
\hline
\end{tabular}

lowing characteristics of the F-QPSK-B signal. Note that the F-QPSK-E ${ }^{2}$ signal has the same characteristics.

1) The F-QPSK-B signal has only five single-phase transition values, i.e., $\pm \pi / 2, \pm \pi / 4$, and 0 .

2) The phase changes linearly during the $T_{\mathrm{b}}$ interval, where the magnitude of the single-phase transition is equal to $\pi / 2$, i.e., $\phi(t)-\phi\left(n T_{\mathrm{b}}\right)=\pi / 2 \cdot\left(t-n T_{\mathrm{b}}\right) / T_{\mathrm{b}}, n T_{\mathrm{b}}<$ $t<(n+1) T_{\mathrm{b}}$.

3) The phase does not change during the $T_{\mathrm{b}}$ interval, where the single-phase transition is equal to zero, i.e., $\phi(t)-$ $\phi\left(n T_{\mathrm{b}}\right)=0, n T_{\mathrm{b}}<t<(n+1) T_{\mathrm{b}}$.

In order to specify $g_{0}(t)$, it is necessary to determine the length and symmetry characteristics of $g_{0}(t)$. If the length of $g_{0}(t)$ having a nonzero value is less than or equal to $2 T_{\mathrm{b}}$, the number of possible single-phase transitions is less than or equal to four. Therefore, it does not satisfy the first characteristic. If the length is greater than $2 T_{\mathrm{b}}$, except for lengths of $3 T_{\mathrm{b}}$ and $4 T_{\mathrm{b}}$, the number of possible single-phase transitions is more 
than five. It also does not satisfy the first characteristic. Hence, the length of $g_{0}(t)$ must be $3 T_{\mathrm{b}}$ or $4 T_{\mathrm{b}}$.

When the length of $g_{0}(t)$ is equal to $4 T_{\mathrm{b}}$, and when the parts of $g_{0}(t)$ separated by the $T_{\mathrm{b}}$ interval are equal, i.e., $\left.g_{0}(t)\right|_{\left(0<t<T_{\mathrm{b}}\right)}=\left.g_{0}(t)\right|_{\left(T_{\mathrm{b}}<t<2 T_{\mathrm{b}}\right)}=\left.g_{0}(t)\right|_{\left(2 T_{\mathrm{b}}<t<3 T_{\mathrm{b}}\right)}=$ $\left.g_{0}(t)\right|_{\left(3 T_{\mathrm{b}}<t<4 T_{\mathrm{b}}\right)}$, the number of possible single-phase transitions becomes equal to five.

In order to also satisfy the second and third characteristics, the premodulation filter must be 4 REC, i.e., $g_{0}(t)=1 /\left(8 T_{\mathrm{b}}\right)$, $0<t<4 T_{\mathrm{b}}$. It does not generate an F-QPSK- ${ }^{2}$ signal. However, when the length of $g_{0}(t)$ is equal to $3 T_{\mathrm{b}}$, and if $g_{0}(t)$ is even symmetric with respect to the line $t=3 T_{\mathrm{b}} / 2$, i.e., $g_{0}(t)=$ $g_{0}\left(3 T_{\mathrm{b}}-t\right)$, then the number of possible single-phase transitions becomes equal to five. Therefore, $g_{0}(t)$ for the CPM of the F-QPSK-E ${ }^{2}$ signal must have the characteristics of symmetry and must have a length equal to $3 T_{\mathrm{b}}$. Additional characteristics of $g_{0}(t)$ can be found by utilizing the first characteristic, which is written as

$$
\int_{k T_{\mathrm{b}}}^{(k+1) T_{\mathrm{b}}} g_{0}(t)= \begin{cases}1 / 4, & \mathrm{k}=1 \\ 1 / 8, & \mathrm{k}=0,2 \\ 0, & \text { otherwise. }\end{cases}
$$

We can now derive the instantaneous frequency of the F-QPSK-E ${ }^{2}$ signal using the second and third characteristics. When the magnitude of the single-phase transition is equal to $\pi / 2$, input binary data bits $\left(w_{n-1}, w_{n}, w_{n+1}\right)$ for the CPM of F-QPSK-E ${ }^{2}$ must be the same, i.e., $(1,1,1)$ or $(-1,-1,-1)$. The instantaneous frequency is then written as [assuming that the input binary data bits are equal to $(1,1,1)$ for the singlephase transition of $+\pi / 2$ ]

$$
\begin{array}{r}
g_{0}\left(t-(n-1) T_{\mathrm{b}}\right)+g_{0}\left(t-n T_{\mathrm{b}}\right)+g_{0}\left(t-(n+1) T_{\mathrm{b}}\right)=\frac{1}{\left(2 T_{\mathrm{b}}\right)} \\
(n+1) T_{\mathrm{b}}<t<(n+2) T_{\mathrm{b}} .
\end{array}
$$

When the magnitude of the single-phase transition is equal to zero, input binary data bits $\left(w_{n-1}, w_{n}, w_{n+1}\right)$ must satisfy that $w_{n-1}$ is the same as $w_{n+1}$, while it is different than $w_{n}$, i.e., $(-1,1,-1)$ or $(1,-1,1)$. The instantaneous frequency is then written as [assuming that the binary data bits are equal to $(-1,1,-1)]$

$$
\begin{array}{r}
-g_{0}\left(t-(n-1) T_{\mathrm{b}}\right)+g_{0}\left(t-n T_{\mathrm{b}}\right)-g_{0}\left(t-(n+1) T_{\mathrm{b}}\right)=0 \\
(n+1) T_{\mathrm{b}}<t<(n+2) T_{\mathrm{b}} .
\end{array}
$$

The sum of (7) and (8) is written as

$$
g_{0}\left(t-n T_{\mathrm{b}}\right)=\frac{1}{\left(4 T_{\mathrm{b}}\right)}, \quad(n+1) T_{\mathrm{b}}<t<(n+2) T_{\mathrm{b}} .
$$

Equation (9) specifies the second $T_{\mathrm{b}}$ interval, i.e., $T_{\mathrm{b}}<t<$ $2 T_{\mathrm{b}}$, of $g_{0}(t)$. Note that (9) satisfies a characteristic of (6).

The first $T_{\mathrm{b}}$ interval of $g_{0}(t)$ can be derived using the instantaneous frequency for the single-phase transition of $\pm \pi / 4$. From the waveforms shown in Table II and [13], the I/Q baseband waveforms for the single-phase transition of $+\pi / 4$ are written as

$$
\begin{aligned}
& I(t)= \cos \left(\frac{\pi}{2 T_{\mathrm{b}}}\left(t-(n+1) T_{\mathrm{b}}\right)\right) \\
&-\left(1-\frac{1}{\sqrt{2}}\right) \cos ^{3}\left(\frac{\pi}{2 T_{\mathrm{b}}}\left(t-(n+1) T_{\mathrm{b}}\right)\right) \\
& Q(t)=1-\frac{1}{2} \cdot \cos ^{2}\left(\frac{\pi}{2 T_{\mathrm{b}}}\left(t-(n+1) T_{\mathrm{b}}\right)\right) \\
&+\left(\frac{1}{\sqrt{2}}-\frac{1}{2}\right) \cdot \cos ^{4}\left(\frac{\pi}{2 T_{\mathrm{b}}}\left(t-(n+1) T_{\mathrm{b}}\right)\right) \\
&(n+1) T_{\mathrm{b}}<t \leq(n+2) T_{\mathrm{b}} .
\end{aligned}
$$

The instantaneous frequency is derived as follows:

$$
\frac{d \phi(t)}{d t}=\frac{d}{d t}\left(\tan ^{-1}\left(\frac{Q(t)}{I(t)}\right)\right)=\frac{Q^{\prime}(t) I(t)-Q(t) I^{\prime}(t)}{I^{2}(t)+Q^{2}(t)} .
$$

Since the F-QPSK-E ${ }^{2}$ signal has a constant envelope (envelope fluctuation of less than $0.03 \mathrm{~dB}$ ), (11) is written as

$$
\frac{d \phi(t)}{d t}=Q^{\prime}(t) I(t)-Q(t) I^{\prime}(t) .
$$

Using (10) and (12), the instantaneous frequency is written as

$$
\begin{aligned}
\frac{d \phi(t)}{d t}=\frac{\pi}{2 \cdot T_{\mathrm{b}}} & \cdot \sin \left(\frac{\pi}{2 \cdot T_{\mathrm{b}}}\left(t-(n+1) T_{\mathrm{b}}\right)\right) \\
\cdot & \left\{1+\left(\frac{3}{\sqrt{2}}-\frac{5}{2}\right) \cdot \cos ^{2}\left(\frac{\pi}{2 \cdot T_{\mathrm{b}}}\left(t-(n+1) T_{\mathrm{b}}\right)\right)\right. \\
+ & \left(2-\frac{7}{2 \sqrt{2}}\right) \cdot \cos ^{4}\left(\frac{\pi}{2 \cdot T_{\mathrm{b}}}\left(t-(n+1) T_{\mathrm{b}}\right)\right) \\
+ & \left.\left(\frac{3-2 \sqrt{2}}{2 \sqrt{2}}\right) \cdot \cos ^{6}\left(\frac{\pi}{2 \cdot T_{\mathrm{b}}}\left(t-(n+1) T_{\mathrm{b}}\right)\right)\right\} \\
& (n+1) T_{\mathrm{b}}<t \leq(n+2) T_{\mathrm{b}} .
\end{aligned}
$$

Since the signal in (10) crosses the $Q$-axis in signal space at $t=(n+2) T_{\mathrm{b}}$, the allowed single-phase transition between times $t=(n+2) T_{\mathrm{b}}$ and $t=(n+3) T_{\mathrm{b}}$ must be $\pi / 4$ or $\pi / 2$ [21]. In order to satisfy the phase transition between times $t=$ $(n+1) T_{\mathrm{b}}$ and $t=(n+3) T_{\mathrm{b}}$, the input binary data bits $\left(w_{n-1}\right.$, $\left.w_{n}, w_{n+1}\right)$ must be equal to $(-1,1,1)$. The instantaneous frequency is then written as

$$
\begin{gathered}
-g_{0}\left(t-(n-1) T_{\mathrm{b}}\right)+g_{0}\left(t-n T_{\mathrm{b}}\right)+g_{0}\left(t-(n+1) T_{\mathrm{b}}\right) \\
=\frac{1}{2 \pi h} \cdot \frac{d \phi(t)}{d t}, \quad(n+1) T_{\mathrm{b}}<t<(n+2) T_{\mathrm{b}} .
\end{gathered}
$$

The subtraction of (8) from (14) is written as

$$
\begin{aligned}
g_{0}( & \left.-(n+1) T_{\mathrm{b}}\right) \\
= & \frac{1}{4 \pi h} \cdot \frac{d \phi(t)}{d t} \\
& =\frac{1}{2 \pi} \cdot \frac{d \phi(t)}{d t}, \quad(n+1) T_{\mathrm{b}}<t<(n+2) T_{\mathrm{b}} .
\end{aligned}
$$




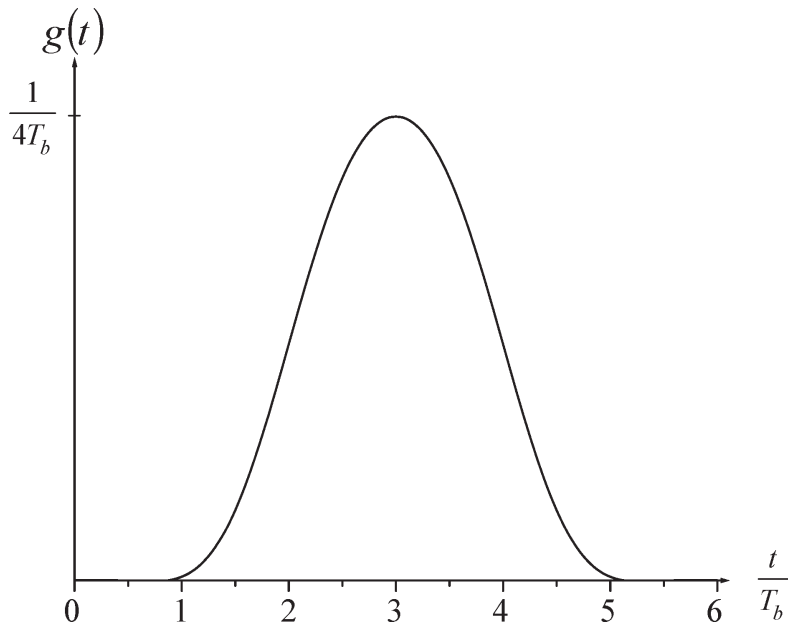

Fig. 6. Proposed premodulation filter for CPM of F-QPSK-B.

Equation (15) specifies the first $T_{\mathrm{b}}$ interval of $g_{0}(t)$. Note that (15) also satisfies a characteristic of (6). The third $T_{\mathrm{b}}$ interval of $g_{0}(t)$ can be derived similarly. Using (9) and (15), the premodulation filter for the CPM of F-QPSK- ${ }^{2} g_{0}(t)$ is written as (16), shown at the bottom of page.

The power spectrum of the F-QPSK-E ${ }^{2}$ signal is a little broader than that of the F-QPSK-B signal. Therefore, an additional LPF is necessary for the premodulation filter for the CPM of F-QPSK-B. The premodulation filter $g(t)$ for the CPM of the F-QPSK-B can be approximated using a cascaded $g_{0}(t)$ and the raised cosine (RC) filter, which is shown in Fig. 6. The chosen roll-off factor and $-6-\mathrm{dB}$ bandwidth of the $\mathrm{RC}$ filter are equal to 1.0 and $0.44 / T_{\mathrm{b}}$, respectively.

\section{D. $A C M D E$}

In the previous sections, the modulation index and the premodulation filter for the CPM of the F-QPSK-B have been specified. However, it is necessary to note that input binary data for I/Q modulation and for CPM of F-QPSK-B are different for the same phase transition behavior. For the four consecutive input binary data bits $\left(q_{n-1}, q_{n}, q_{n+1}, q_{n+2}\right)$ in the I/Q modulation of F-QPSK-B, if $q_{n-1}$ is equal to $q_{n+1}$ and $q_{n}$ is equal to $q_{n+2}$, e.g., $(0,0,0,0),(1,0,1,0)$, the corresponding single-phase transition will be equal to zero. If $q_{n-1}$ is equal to $q_{n+1}$ and $q_{n}$ is not equal to $q_{n+2}$, and vice versa, e.g., $(1,0,1,1),(1,0$, 0,0 ), the corresponding single-phase transition will be equal to
TABLE III

RELATIONSHIP BETWEEN INPUT DATA AND SINGLE-PHASE TRANSITION OF I/Q MODULATED F-QPSK-B AND CONTINUOUS-PHASE-MODULATED F-QPSK-B SIGNAL

\begin{tabular}{|c|c|c|}
\hline $\begin{array}{c}\text { Single-phase } \\
\text { transition of } \\
\text { modulated signal }\end{array}$ & $\begin{array}{c}\text { Input data } \\
\left(q_{n-1}, q_{n}, q_{n+1}, q_{n+2}\right) \\
\text { for I/Q modulation of } \\
\text { F-QPSK-B }\end{array}$ & $\begin{array}{c}\text { Input data } \\
\left(w_{n-1}, w_{n}, w_{n+1}\right) \\
\text { for CPM of } \\
\text { F-QPSK-B } \\
\end{array}$ \\
\hline 0 & $\begin{array}{l}(0,0,0,0),(0,1,0,1) \\
(1,0,1,0),(1,1,1,1)\end{array}$ & $(0,1,0),(1,0,1)$ \\
\hline$+\pi / 4,-\pi / 4$ & $\begin{array}{l}(0,0,0,1),(0,1,0,0) \\
(0,0,1,0),(0,1,1,1) \\
(1,0,1,1),(1,1,1,0) \\
(1,0,0,0),(1,1,0,1)\end{array}$ & $\begin{array}{l}(0,1,1),(1,1,0), \\
(0,0,1),(1,0,0)\end{array}$ \\
\hline$+\pi / 2,-\pi / 2$ & $\begin{array}{l}(0,0,1,1),(0,1,1,0) \\
(1,0,0,1),(1,1,0,0)\end{array}$ & $(1,1,1),(0,0,0)$ \\
\hline
\end{tabular}

$\pi / 4$ or $-\pi / 4$. Also, if $q_{n-1}$ is not equal to $q_{n+1}$ and $q_{n}$ is not equal to $q_{n+2}$, e.g., $(1,0,0,1),(0,1,1,0)$, the corresponding single-phase transition will be equal to $\pi / 2$ or $-\pi / 2$.

However, for the three consecutive input binary data bits $\left(w_{n-1}, w_{n}, w_{n+1}\right)$ in the CPM of the F-QPSK-B, $w_{n-1}$ must be equal to $w_{n+1}$ while not being equal to $w_{n}$, e.g., $(1,0,1)$, $(0,1,0)$ for the zero single-phase transition. For the singlephase transition of $\pi / 4$ or $-\pi / 4, w_{n-1}$ must not be equal to $w_{n+1}$, e.g., $(1,1,0),(0,0,1)$. Also, $w_{n-1}, w_{n}$, and $w_{n+1}$ must be equal for the single-phase transition of $\pi / 2$ or $-\pi / 2$.

Table III compares the input binary data for the I/Q modulation of the F-QPSK-B to the input binary data for the CPM of the F-QPSK-B for the same single-phase transition. Table III shows that consecutive input data bits for the CPM of the F-QPSK-B must be different, i.e., $w_{n-1} \neq w_{n}$, to have the same single-phase transition as the I/Q modulation of the F-QPSK-B, in which the alternating input data bits for the $\mathrm{I} / \mathrm{Q}$ modulation of the F-QPSK-B are the same, i.e., $q_{n-1}=q_{n+1}$, and vice versa. This means that, using a differential encoder, the continuousphase-modulated F-QPSK-B signal can have the same phase transition behavior as the I/Q modulated F-QPSK-B signal. In this paper, we propose an ACMDE, which is written as

$$
\begin{aligned}
& w_{n}=w_{n-1} \oplus \overline{\left(q_{n-1} \oplus q_{n+1}\right)}, \quad n=1,2, \ldots \\
& w_{0}=q_{0} \oplus q_{1}
\end{aligned}
$$

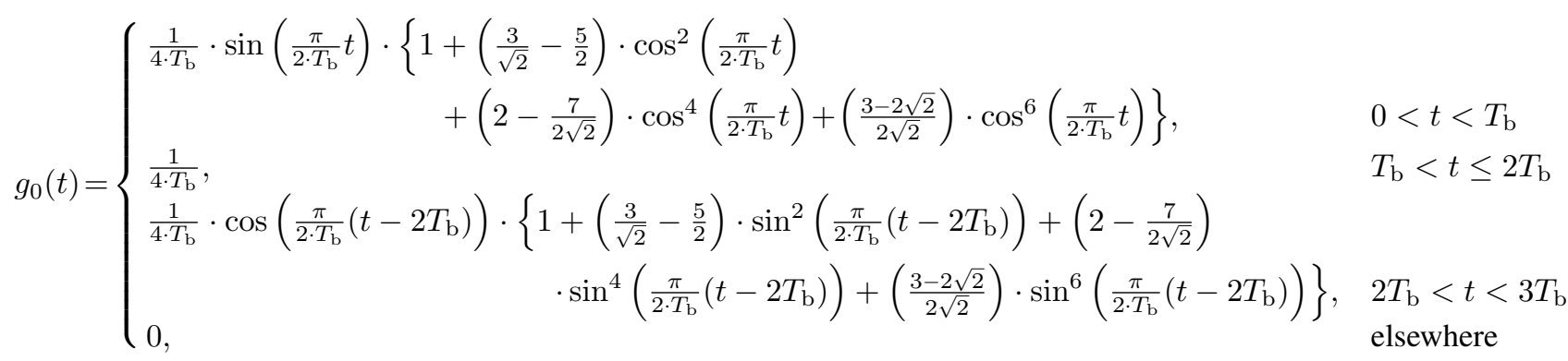




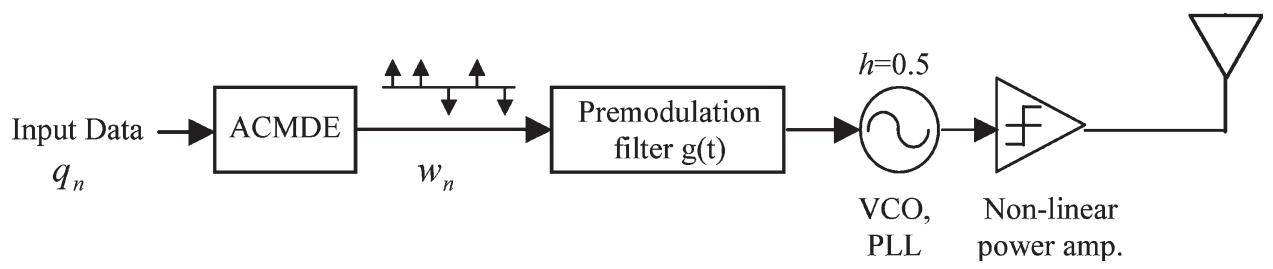

Fig. 7. ACMDE + CPM based F-QPSK-B modulation architecture. Note that it produces basically the same modulated signal as I/Q modulation of F-QPSK-B.

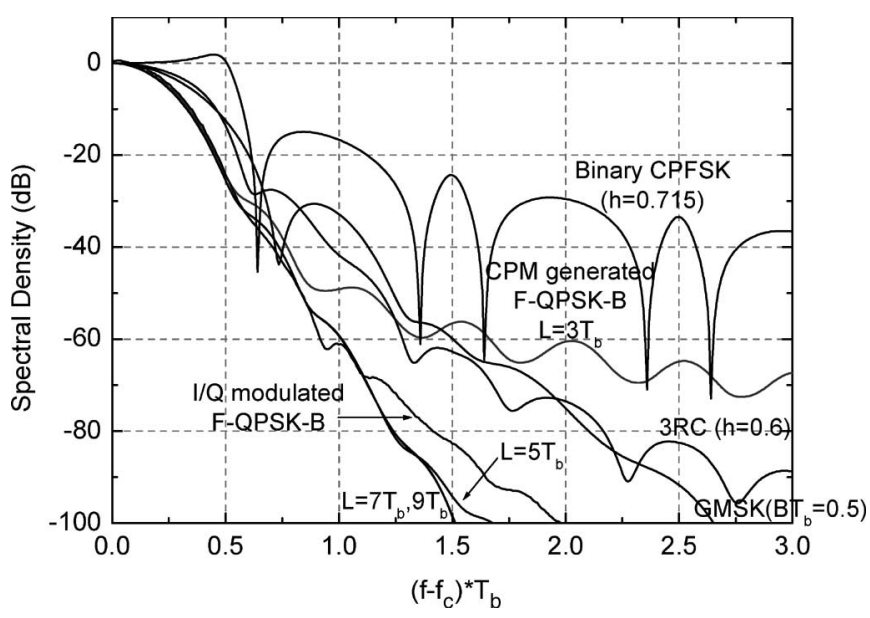

Fig. 8. PSD of the continuous-phase-modulated F-QPSK-B signal with different truncation lengths of the premodulation filter $g(t)$.

where $q_{i}$ denotes the input data both for the I/Q modulation of the F-QPSK-B and for the CPM of the F-QPSK-B. ACMDE output data is denoted by $w_{i}$, which is used as the input data for the premodulation filter for the CPM of the F-QPSK-B. $\oplus$ refers to modulo- 2 addition. Fig. 7 shows a block diagram of the ACMDE + CPM-based modulation architecture of the F-QPSK-B. This architecture produces basically the same modulated signal as is produced by the I/Q modulation of the F-QPSK-B, which is shown in Fig. 1(a) and (h). Further note that an ACMDE can improve the BER by two times over uncoded data because it allows direct symbol-by-symbol coherent detection. This does not require decoding for the encoding effect inherent in all CPM. ${ }^{3}$

\section{PERformance AnAlysis}

\section{A. Performance Comparison of CPM of F-QPSK-B With $I / Q$ Modulation of F-QPSK-B}

Fig. 8 shows the PSD of the proposed continuous-phasemodulated F-QPSK-B signal, taking the truncation length of $g(t) L$ as a parameter. In order to calculate the PSDs, except that of the I/Q modulated F-QPSK-B signal [14], the method proposed by Anderson and Salz [22] is used. In Fig. 8, it is shown that the continuous-phase-modulated F-QPSK-B signal,

\footnotetext{
${ }^{2}$ For calculations in the premodulation filter, 1 is mapped to 1 , and 0 is mapped to -1 .

${ }^{3}$ Some CPM signal with a modulation index of 0.5 requires a differential decoder for the coherent symbol-by-symbol detection [29], [31]. ACMDE is used as the differential decoder for the coherent symbol-by-symbol detection of the uncoded CPM of F-QPSK-B. However, the ACMDE in the receiver causes a two-bit error for a one-bit input error.
}

in which $L$ is longer than or equal to $5 T_{\mathrm{b}}$, has practically the same PSD as the I/Q modulated F-QPSK-B signal. Fig. 9 shows that the eye diagrams of the continuous-phase-modulated and I/Q modulated F-QPSK-B signals are almost the same.

Fig. 10 shows the union bound and simulated BER performance of the optimum coherent detection (see Appendix B) of the CPM of the F-QPSK-B in the adaptive white Gaussian noise (AWGN) channel. Since the number of waveforms for "1" and " 0 " is equal to 64, and because each waveform is equiprobable, the union bound of the F-QPSK-B signal is written as

$$
P_{\mathrm{e}} \leq \frac{1}{64} \sum_{i=0}^{63}\left(\sum_{j=0}^{63} Q\left(\sqrt{d_{i j}^{2} \frac{E_{\mathrm{b}}}{N_{0}}}\right)\right)
$$

where $Q(x)$ is defined as

$$
Q(x)=\frac{1}{\sqrt{2 \pi}} \int_{x}^{\infty} e^{-t^{2} / 2} d t .
$$

When $E_{\mathrm{b}} / N_{0}$ is large, the union bound becomes tight and is dominated by the minimum normalized squared Euclidean distance (MNSED) $d_{\min }^{2}$ [23]. Hence, the approximated union bound of the F-QPSK-B signal is written as

$$
P_{\mathrm{e}} \approx C \cdot Q\left(\sqrt{d_{\mathrm{min}}^{2} \cdot \frac{E_{\mathrm{b}}}{N_{0}}}\right)
$$

where error coefficient $C$ is equal to 0.56 for the F-QPSK-B signal. In the continuous-phase-modulated F-QPSK-B signal, $d_{\min }^{2}$, which is calculated using the method proposed by Aulin et al. [24], is equal to 1.50 when $L$ is equal to or greater than $5 T_{\mathrm{b}}$.

Fig. 10 also shows the approximated union bound for the continuous-phase-modulated F-QPSK-B signal. As $E_{\mathrm{b}} / N_{0}$ increases, the union bound becomes the same as the approximated union bound and approaches the simulation result. This means that the union bound is a tight upper bound for optimum coherent detection of the continuous-phase-modulated F-QPSK-B signal.

Fig. 10 shows that the union bound and simulation result of the continuous-phase-modulated F-QPSK-B signal have only about $0.1-\mathrm{dB}$ performance degradation as compared to the I/Q modulated F-QPSK-B signal. ${ }^{4}$ This performance degradation is caused by a reduced MNSED of the continuousphase-modulated F-QPSK-B signal. In Section III-C and D, it has been shown that three consecutive input binary data bits

\footnotetext{
${ }^{4}$ Appendix A represents the MNSED of the I/Q modulated F-QPSK-B signal.
} 


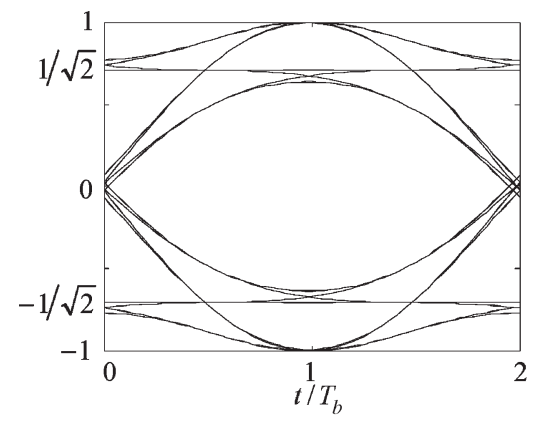

(a)

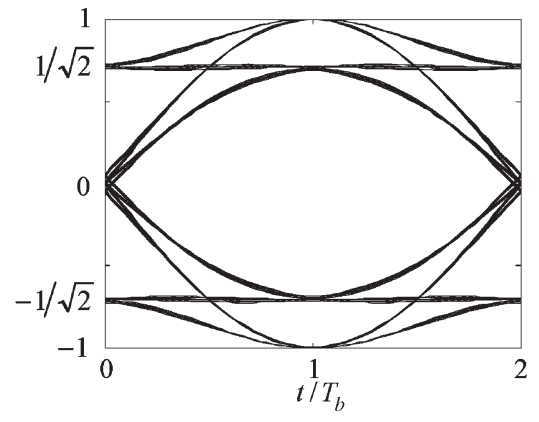

(b)

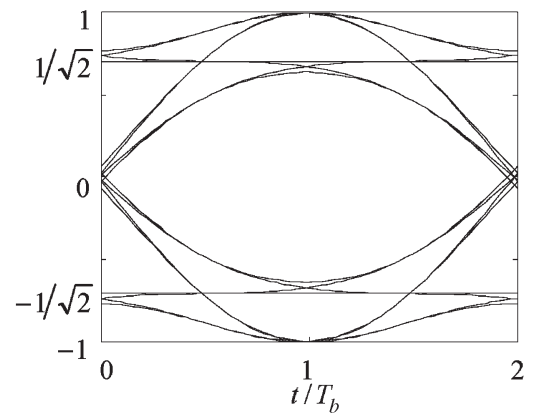

(c)

Fig. 9. Simulated eye diagrams of F-QPSK-B signal (a) CPM $(h=0.5)$, (b) I/Q modulation $(A=1 / \sqrt{2})$, and (c) AMP decomposition.

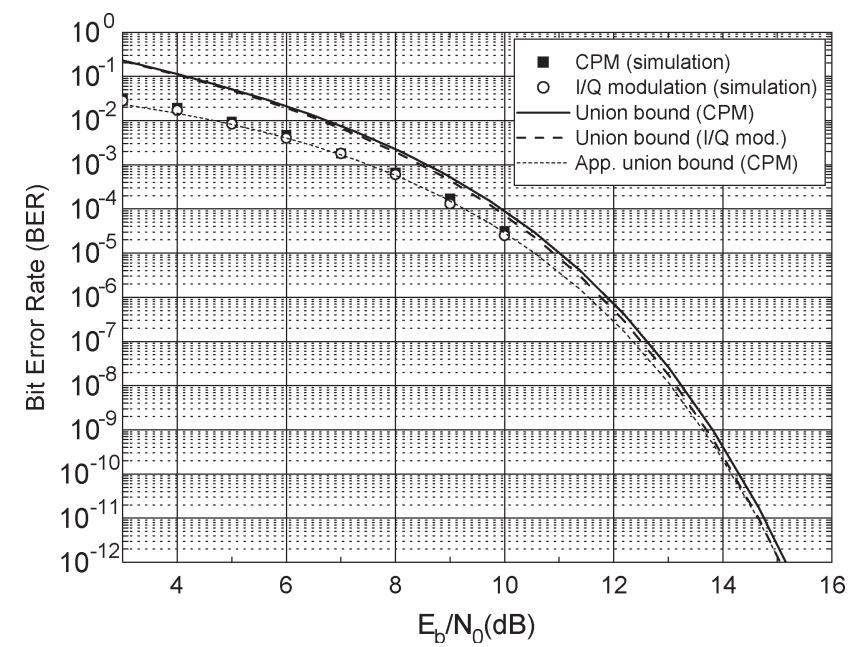

Fig. 10. Union bound and simulated BER performance of optimum coherent detection of CPM of F-QPSK-B. Note that CPM of F-QPSK-B has only about 0.1-dB degraded performance compared to the I/Q modulation of F-QPSK-B.

$\left(w_{n-1}, w_{n}, w_{n+1}\right)$ in the CPM of the F-QPSK-B determine a single-phase transition. However, it has also been shown that to attain the same PSD as is attained when using the I/Q modulated F-QPSK-B signal, the truncation length of $g(t)$ for the CPM of the F-QPSK-B signal must be at least $5 T_{\mathrm{b}}$. When the truncation length of $g(t)$ is equal to $5 T_{\mathrm{b}}$, the phase distribution of $g(t)$ in each $T_{\mathrm{b}}$ interval is equal to $1.3^{\circ}\left(0<t<T_{\mathrm{b}}\right)$, $22.4^{\circ}\left(1 T_{\mathrm{b}}<t<2 T_{\mathrm{b}}\right), 42.6^{\circ}\left(2 T_{\mathrm{b}}<t<3 T_{\mathrm{b}}\right), 22.4^{\circ}\left(3 T_{\mathrm{b}}<\right.$ $\left.t<4 T_{\mathrm{b}}\right), 1.3^{\circ}\left(4 T_{\mathrm{b}}<t<5 T_{\mathrm{b}}\right)$, respectively. This means that, although the effect is small, binary data bits $w_{n-2}$ and $w_{n+2}$ affect a single-phase transition during the $T_{\mathrm{b}}$ interval between times $t=(n+1) T_{\mathrm{b}}$ and $t=(n+2) T_{\mathrm{b}}$. If the binary data bit $w_{n-2}$ or $w_{n+2}$ is different from $w_{n}$, the corresponding singlephase transition of the continuous-phase-modulated F-QPSK-B signal becomes smaller than the one associated with the I/Q modulated F-QPSK-B signal. Therefore, the MNSED of the continuous-phase-modulated F-QPSK-B signal is a little less than that of the I/Q modulated F-QPSK-B signal.

It is useful to compare the BER performance of the continuous-phase-modulated and I/Q modulated F-QPSK-B signals with coherent detection using a simple LPF. One of the advantages of F-QPSK-B is that the F-QPSK-B signal can be coherently detected with a simple LPF, even though the F-QPSK-B signal utilizes a controlled ISI to improve bandwidth efficiency. Since the CPM of F-QPSK-B is a partial response binary $\mathrm{CPM}$ with a modulation index of 0.5 , the BER analysis method introduced by Aulin et al. [25] can be used for symbol-by-symbol coherent detection using an LPF of the continuous-phase-modulated F-QPSK-B signal. Since ACMDE is used in the transmitter, the phase node error probability is equal to the bit error probability. In [25], the phase node error probability $P_{\theta}$ has been defined as

$$
P_{\theta}=\frac{1}{m} \sum_{i=0}^{m-1} Q\left(\sqrt{d_{i}^{2} \frac{E_{\mathrm{b}}}{N_{0}}}\right)
$$

where the normalized squared Euclidean distance $d_{i}^{2}$ and $m$ are written as

$$
\begin{aligned}
& d_{i}^{2}=\frac{2}{T_{\mathrm{b}}} \frac{\left\{\int_{0}^{N_{T} \cdot T_{\mathrm{b}}} h_{r x}(t) \cdot \cos \phi\left(t, \mathbf{w}_{i}\right) d t\right\}^{2}}{\int_{0}^{N_{T} \cdot T_{\mathrm{b}}}\left(h_{r x}(t)\right)^{2} d t} \\
& m=2^{N_{T}+L-1}
\end{aligned}
$$




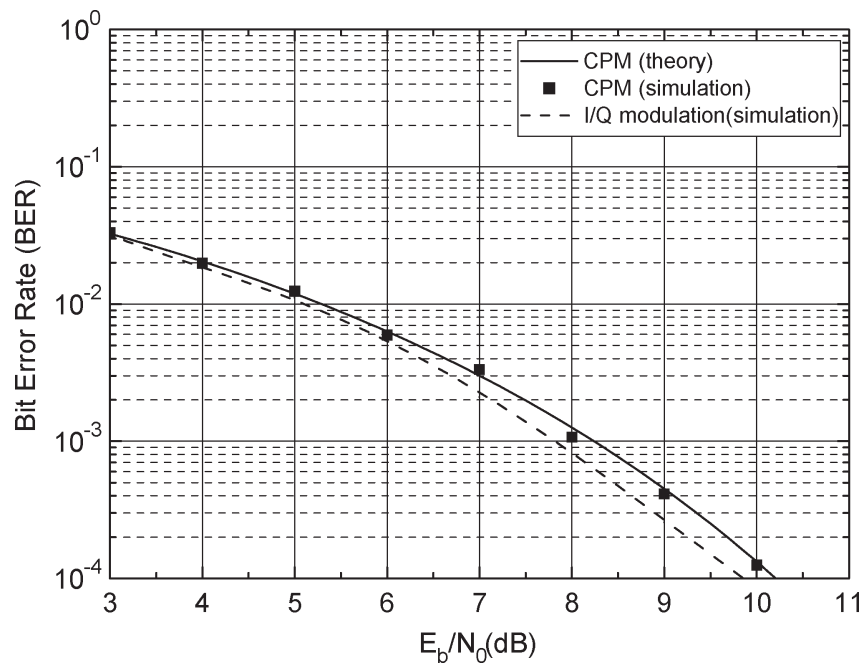

Fig. 11. Calculated and simulated BER performance of symbol-by-symbol coherent detection using an LPF of CPM of F-QPSK-B.

where $h_{r x}(t)$ denotes the impulse response of the receiver filter, in which a fourth-order phase equalized Butterworth filter $\left(-3-\mathrm{dB}\right.$ bandwidth $\left.=0.275 / T_{\mathrm{b}}\right)$ is used. $N_{T}$ denotes the length of the receiver filter impulse response normalized to $T_{\mathrm{b}}$. $N_{T}$ is equal to 12 for the Butterworth filter. $L$ denotes the truncation length of the premodulation filter, normalized to $T_{\mathrm{b}}$, for the CPM of the F-QPSK-B, in which 5 is chosen. Also, $\cos \phi\left(t, \mathbf{w}_{i}\right)$ denotes the cosine value of the modulated phase with binary data sequence $\mathbf{w}_{i}$. Fig. 11 shows calculated and simulated BER performances of symbol-by-symbol coherent detection using an LPF of the CPM of the F-QPSK-B in the AWGN channel. In Fig. 11, it is shown that the calculation result is the same as the simulation result. Fig. 11 also shows that the continuous-phase-modulated F-QPSK-B signal produces a BER of $10^{-4}$ at $E_{\mathrm{b}} / N_{0}$ of $10.2 \mathrm{~dB}$. It suffers only 0.3- $\mathrm{dB}$ degradation at a BER of $10^{-4}$ from the performance of the I/Q modulated F-QPSK-B signal. This is because the MNSED of the Butterworth filtered signal of the continuousphase-modulated F-QPSK-B signal is about $0.2 \mathrm{~dB}$ less than the one of the I/Q modulated F-QPSK-B signal. ${ }^{5}$

It has been shown that the F-QPSK-B signal can be noncoherently detected [21]. Furthermore, it has been shown that BER performance of noncoherent detection can be improved by increasing the received signal's observation time over multisymbols as well as by adopting trellis demodulation. For the limiter discriminator integrate-and-dump (LD I\&D) detection, when the number of symbols observed is equal to $\mathrm{N}$, the number of allowed vectors formed by consecutive LD I\&D detector output signals is equal to $2^{(N+2)}-1$. In the trellis decoding of LD I\&D detection, the allowed vector $\mathbf{c}_{i}$ becomes the $i$ th state of the trellis. Therefore, the number of states is equal to $2^{(N+2)}-1$. Let $c_{i, n}(n=1,2, \ldots, N ; i=1,2, \ldots$, $\left.2^{(N+2)}-1\right)$ denote the $n$th element of the $i$ th allowed vector, and let $r_{k}$ denote the LD I\&D detector output sampled at

\footnotetext{
${ }^{5}$ The CPM characteristics of the F-QPSK-B signal are used to calculate the MNSED of the Butterworth filtered signal of the I/Q modulated F-QPSK-B signal.
}

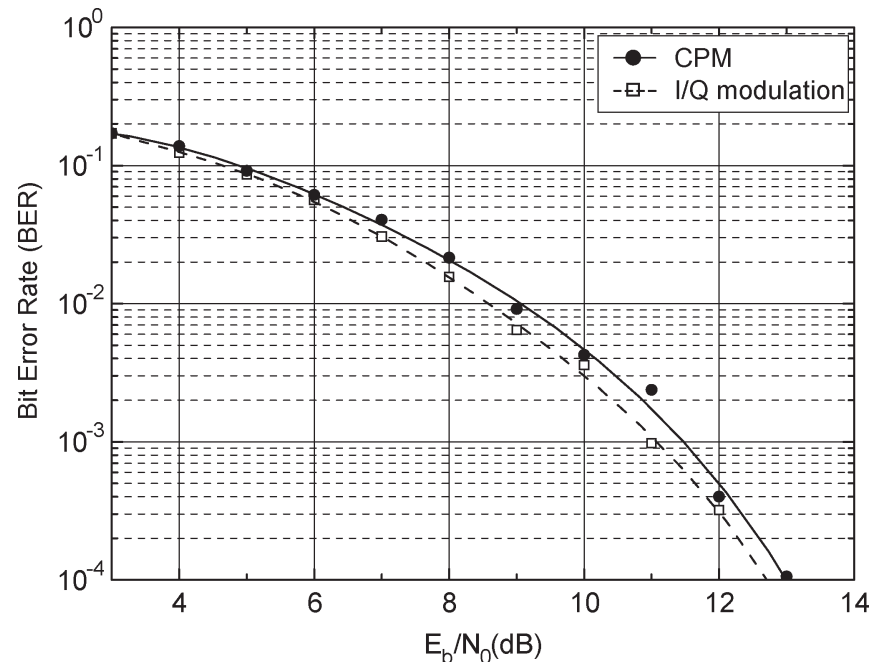

Fig. 12. Simulated BER performance of CPM of F-QPSK-B with LD I\&D followed by trellis decoding with five-symbol observation.

$t=k T_{\mathrm{b}}$. The branch metric of the $i$ th state at time $t=k T_{\mathrm{b}}$ is written as

$$
d_{i, k}=\sum_{n=1}^{N}\left(c_{i, n}-r_{(k+n-(N+1) / 2)}\right)^{2} .
$$

The survival path is the path that has the smallest accumulated branch metric, i.e., state metric. The decoder output is the $(N+1) / 2$ th element of a state, i.e., $c_{i,(N+1) / 2}$, in the survival path. When the magnitude of the sum of two consecutive decoder outputs is larger than or equal to $\pi / 4$, the receiver decides that a "1" was sent. Otherwise, it decides that a "0" was sent. Fig. 12 shows the simulated BER performance of the CPM of F-QPSK-B with LD I\&D followed by trellis decoding with fivesymbol observation in the AWGN channel. It produces a BER of $10^{-4}$ at $E_{\mathrm{b}} / N_{0}$ of $13.0 \mathrm{~dB}$, which has a performance degradation of $0.3 \mathrm{~dB}$ at a BER of $10^{-4}$ from the I/Q modulation of F-QPSK-B.

The CPM characteristics of the F-QPSK-B signal make it possible to use the architecture proposed by Laurent [26] to implement the modulator of the F-QPSK-B signal. In [26], Laurent proposed that a binary CPM signal can be generated as a sum of a finite number of time limited amplitude modulated pulses (AMP). Fig. 13 presents signal components $C_{K}(t)$ $(K=0,1,2, \ldots, 15)^{6}$ of the continuous-phase-modulated F-QPSK-B signal for AMP decomposition. Fig. 9 shows that the eye diagram of the F-QPSK-B signal using the AMP decomposition is the same as that of the continuous-phasemodulated and I/Q modulated F-QPSK-B signals. Even though the AMP decomposition-based F-QPSK-B modulator has more gates than the I/Q modulator, it can have less gates than the continuous phase modulator. Let $N_{s}$ denote the number of samples per input binary data on the AMP decomposition of the F-QPSK-B signal. In addition, let $N_{\mathrm{p}}$ denote the bit width of the address for the waveform lookup table on the conventional continuous phase modulator. If $N_{s}$ satisfies the

\footnotetext{
${ }^{6}$ When the truncation length of $g(t)$ for the CPM of the F-QPSK-B is chosen
} to be $5 T_{\mathrm{b}}, K$ must be an integer between 0 and 15 (see [28, eq. 10]). 

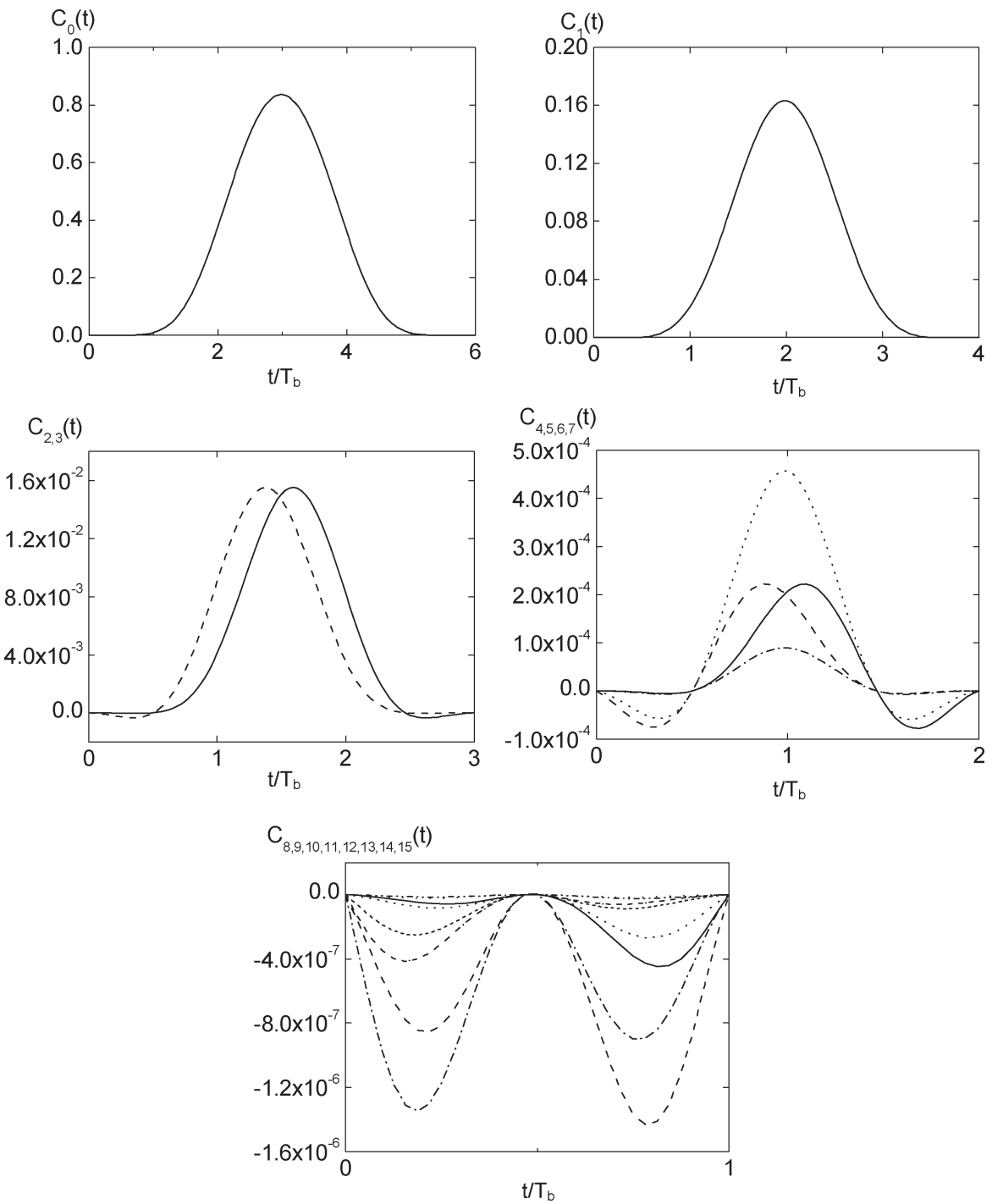

Fig. 13. Signal component functions of CPM of F-QPSK-B for AMP decomposition.

condition of (24), the modulator based on Laurent's method has the advantage over conventional methods in terms of gate size

$$
\log _{2} N_{s} \leq N_{\mathrm{p}}-5
$$

For example, when $N_{s}$ is equal to eight, the modulator based on Laurent's method has a smaller gate size if $N_{\mathrm{p}}$ is more than 8 bits.

In addition, in [27], Kaleh showed that a receiver for CPM signals can be simplified utilizing the AMP decomposition. For the binary CPM signals with a modulation index of 0.5 , Kaleh also proposed the optimum receiver filter for a simple MSK-type receiver by utilizing the AMP decomposition. Since the F-QPSK-B signal is a binary CPM with a modulation index of 0.5 , the receiver for the F-QPSK-B signal can be simplified with an MSK-type receiver, as shown in Appendix C. In Appendix C, the optimum receiver filter for the MSK-type receiver of the F-QPSK-B signal is derived. Fig. 14 shows the BER performances of the CPM of F-QPSK-B with the MSKtype receiver. In Fig. 14, it is shown that the simulated BER lies between the upper and lower bounds. It performs a BER of $10^{-4}$

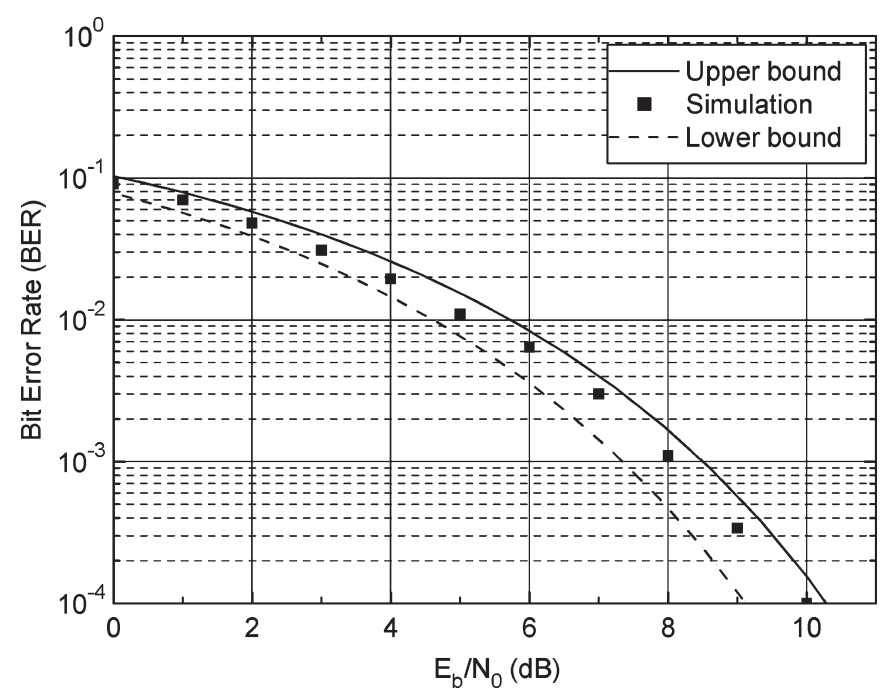

Fig. 14. BER performances of CPM of F-QPSK-B with the MSK-type receiver. 
at $E_{\mathrm{b}} / N_{0}$ of $10.0 \mathrm{~dB}$, which gives a performance degradation of about $0.7 \mathrm{~dB}$ at a BER of $10^{-4}$ from the optimum coherent detection of the CPM of F-QPSK-B, which is shown in Fig. 10.

\section{B. Performance Comparison of CPM of F-QPSK-B With Representative CPM Schemes}

Fig. 8 also compares the PSD of the CPM of F-QPSK-B with that of several CPM schemes such as the optimum binary continuous phase frequency-shift keying (CPFSK) with a modulation index of 0.715 , the GMSK with $B T_{\mathrm{b}}$ of 0.5 , and CPM using an RC premodulation filter. In Fig. 8, it is shown that the CPM of F-QPSK-B achieves a better bandwidth efficiency than the selected CPM schemes.

In [16], Simon compared the throughput and BER performance of the modulations specified for space communication. Utilizing Simon's work, Fig. 15 compares the throughput and the BER performance of the CPM of F-QPSK-B with representative CPM schemes, which include the optimum binary CPFSK with a modulation index of $0.715,{ }^{7}$ the optimum quaternary CPFSK with a modulation index of $0.8,{ }^{8}$ the GMSK [31] with $B T_{\mathrm{b}}$ of 0.25 and 0.5 , CPM using an RC premodulation filter with a length of $2 T_{\mathrm{b}}$ and with a modulation index of 0.6 and 0.8 , and CPM using an RC premodulation filter with a length of $3 T_{\mathrm{b}}$ and with a modulation index of 0.6 and 0.8 . In Fig. 15 , the $x$-axis represents the $E_{\mathrm{b}} / N_{0}$ required for a BER of $10^{-3}$ [Fig. 15(a)], $10^{-4}$ [Fig. 15(b)], and the $y$-axis denotes the reciprocal of the throughput. Note that the $E_{\mathrm{b}} / N_{0}$ is obtained by using the optimum coherent receiver [20]. The throughput is defined as the reciprocal ratio of the frequency band around the carrier frequency containing $99 \%$ of the signal power to the data rate. In order to calculate the $99 \%$ bandwidth, the analytical calculation of the PSD is used [22]. Note that the F-QPSK-B achieves the higher throughput than the selected CPM schemes without the loss of much power efficiency.

\section{CONClusion}

In this paper, the CPM method for F-QPSK-B has been proposed for the first time. The CPM characteristics of the F-QPSK-B signal have been derived from the quasiconstant envelope and continuous phase characteristics of the F-QPSK-B signal.

The modulation index for CPM of F-QPSK-B has been derived from the maximum single-phase transition of the F-QPSK-B signal. The premodulation filter has been derived from the single-phase transition and transient phase characteristics. The ACMDE, which is a differential encoder, has also been proposed so that the continuous-phase-modulated F-QPSK-B signal can have the same phase behavior as the I/Q modulated F-QPSK-B signal.

It has been shown that the PSD and eye diagram of the continuous-phase-modulated F-QPSK-B signal are practically the same as those of the I/Q modulated F-QPSK-B signal.

\footnotetext{
${ }^{7}$ In [28] and [29], it was shown that the optimum modulation index for the binary CPFSK is equal to 0.715 .

${ }^{8}$ In [30], Schonhoff showed that for the quaternary CPFSK, a modulation index of 0.8 minimizes the probability of error.
}

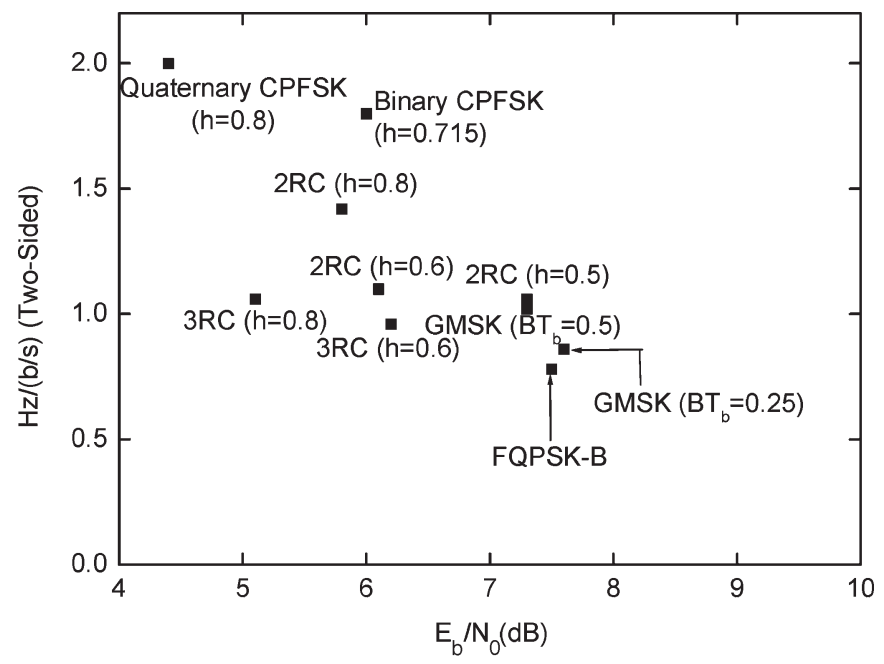

(a)

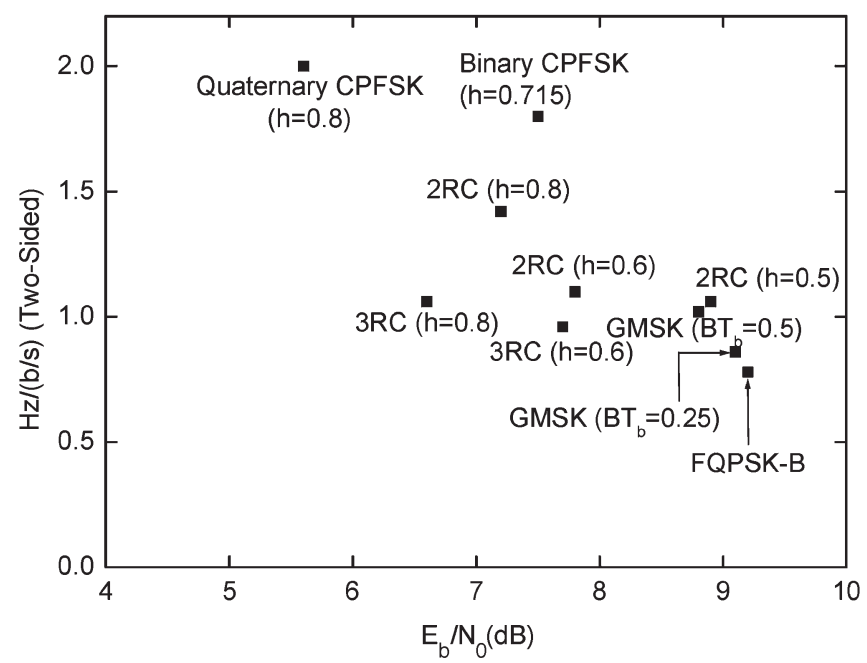

(b)

Fig. 15. Power-bandwidth efficiency comparison of CPM of F-QPSK-B and representative CPM schemes. The $x$-axis represents the $E_{\mathrm{b}} / N_{0}$ required for (a) a BER of $10^{-3}$ and (b) a BER of $10^{-4}$.

Theoretical analysis and simulation results have shown that the continuous-phase-modulated F-QPSK-B signal has almost the same BER performance as the I/Q modulation of F-QPSK-B.

\section{APPENDIX A}

\section{MNSED OF THE I/Q MODULATED F-QPSK-B SIGNAL}

In order to calculate the MNSED of the I/Q modulated F-QPSK-B signal, it is necessary to examine the phase behavior of the F-QPSK-B signal. From Section III-A, we can find that input data $q_{n}$ affects three phase points $\phi_{n-1}, \phi_{n}$, and $\phi_{n+1}$, and the phase transition during $4 T_{\mathrm{b}}$ between times $t=(n-2) T_{\mathrm{b}}$ and $t=(n+2) T_{\mathrm{b}}$, which is shown in Fig. 16. Therefore, the MNSED of the I/Q modulated F-QPSK-B signal $d_{\min }^{2}$ is written as

$$
d_{\min }^{2}=\frac{1}{2 E_{\mathrm{b}}} \int_{(n-2) T_{\mathrm{b}}}^{(n+2) T_{\mathrm{b}}}\left(s\left(t, \mathbf{q}_{\alpha}\right)-s\left(t, \mathbf{q}_{\beta}\right)\right)^{2} d t
$$




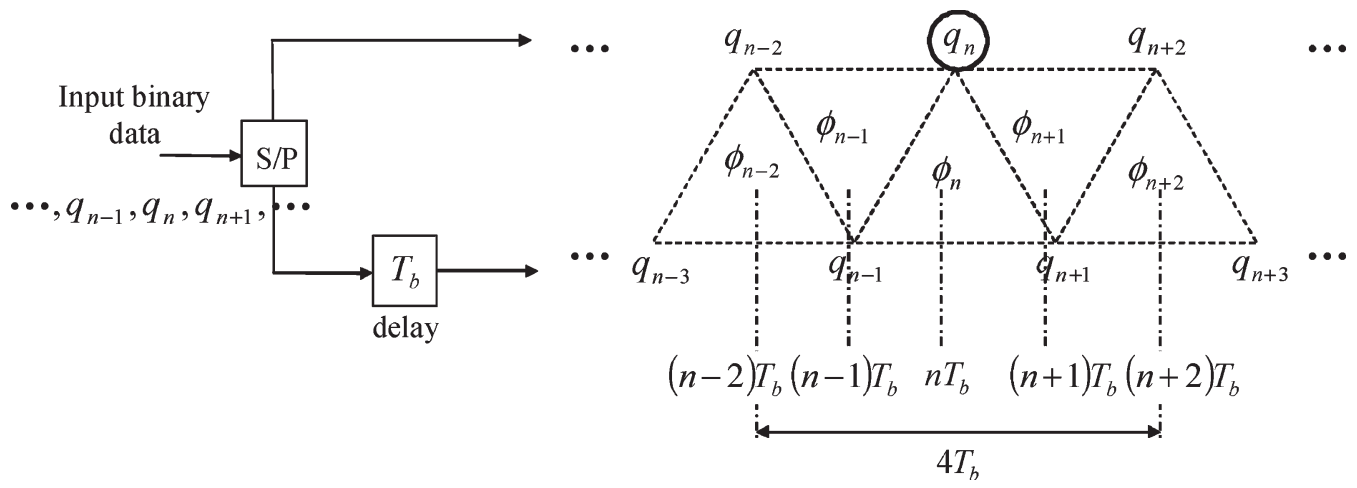

Fig. 16. Phase transition characteristics of the F-QPSK-B signal.

where $\mathbf{q}_{\alpha}$ is equal to $\left(\ldots, q_{n-1}, 1, q_{n+1}, \ldots\right)$, and $\mathbf{q}_{\beta}$ is equal to $\left(\ldots, q_{n-1}, 0, q_{n+1}, \ldots\right)$. The calculated MNSED of the I/Q modulated F-QPSK-B signal is equal to 1.53.

\section{APPENDIX B \\ OPTIMUM COHERENT RECEIVER OF THE F-QPSK-B SIGNAL}

The optimum coherent receiver of the F-QPSK-B signal is the ML receiver, which is based on the distance characteristic presented in Appendix A. Since seven binary data bits are necessary for phase transition during $4 T_{\mathrm{b}}$ (see Fig. 16), the optimum coherent receiver has 128-matched filters. Since the phase points determined by the input data sequence $\left(I_{n}, Q_{n}, I_{n+1}\right)$ and $\left(Q_{n-1}, I_{n}, Q_{n}\right)$ are different, as shown in Section III-A, two 128-matched filter banks are required. However, each bank must be used alternately at every $T_{\mathrm{b}}$.

Let the received signal be given by

$$
r(t)=s\left(t, \mathbf{q}_{i}\right)+n(t), \quad i=\{0,1, \ldots, 127\} .
$$

Each branch calculates the Euclidean distance between $r(t)$ and $s\left(t, \mathbf{q}_{j}\right)$

$$
\begin{aligned}
d_{j}^{2} & =\int_{0}^{4 T_{\mathrm{b}}}\left[r(t)-s\left(t, \mathbf{q}_{j}\right)\right]^{2} d t, \quad j=\{0,1, \ldots, 127\} \\
& =\int_{0}^{4 T_{\mathrm{b}}} r^{2}(t) d t+\int_{0}^{4 T_{\mathrm{b}}} s^{2}\left(t, \mathbf{q}_{j}\right) d t-2 \int_{0}^{4 T_{\mathrm{b}}} r(t) s\left(t, \mathbf{q}_{j}\right) d t .
\end{aligned}
$$

Since the first term in (B.2) is independent of $\mathbf{q}_{j}$ and the F-QPSK-B signal has a constant envelope, the Euclidean distance between $r(t)$ and $s\left(t, \mathbf{q}_{j}\right)$ is determined by correlating $r(t)$ and $s\left(t, \mathbf{q}_{j}\right)$. Therefore, the correlator output of the $j$ th branch of the I/Q channel data is written as

$$
\begin{aligned}
C_{j,\{I, Q\}}=\int_{0}^{4 T_{\mathrm{b}}} r(t) \cos \left(\omega_{\mathrm{o}} t+\theta\left(t, \mathbf{q}_{j,\{I, Q\}}\right)\right) d t \\
\\
j=\{0,1, \ldots, 127\} .
\end{aligned}
$$

When the noise $n(t)$ is written as

$$
n(t)=n_{I}(t) \cdot \cos \left(\omega_{\mathrm{o}} t\right)-n_{Q}(t) \cdot \sin \left(\omega_{\mathrm{o}} t\right)
$$

$r(t)$ is then written as

$$
\begin{aligned}
r(t)= & \cos \left(\omega_{\mathrm{o}} t+\theta\left(t, \mathbf{q}_{i,\{I, Q\}}\right)\right)+n(t), \quad i=\{0,1, \ldots, 127\} \\
= & \left(\cos \left(\theta\left(t, \mathbf{q}_{i,\{I, Q\}}\right)\right)+n_{I}(t)\right) \cos \left(\omega_{\mathrm{o}} t\right) \\
& -\left(\sin \left(\theta\left(t, \mathbf{q}_{i,\{I, Q\}}\right)\right)+n_{Q}(t)\right) \sin \left(\omega_{\mathrm{o}} t\right) \\
= & r_{I}(t) \cdot \cos \left(\omega_{\mathrm{o}} t\right)-r_{Q}(t) \cdot \sin \left(\omega_{\mathrm{o}} t\right)
\end{aligned}
$$

where $r_{I}(t)$ and $r_{Q}(t)$ denote $\left(\cos \left(\theta\left(t, \mathbf{q}_{i,\{I, Q\}}\right)\right)+n_{I}(t)\right)$ and $\left(\sin \left(\theta\left(t, \mathbf{q}_{i,\{I, Q\}}\right)\right)+n_{Q}(t)\right)$, respectively.

By inserting (B.5) in (B.3) and omitting high-frequency terms, it is written as

$$
\begin{gathered}
C_{j,\{I, Q\}}=\int_{0}^{4 T_{\mathrm{b}}}\left\{r_{I}(t) \cdot \cos \left(\theta\left(t, \mathbf{q}_{j,\{I, Q\}}\right)\right)\right. \\
\left.+r_{Q}(t) \cdot \sin \left(\theta\left(t, \mathbf{q}_{j,\{I, Q\}}\right)\right)\right\} d t \\
j=\{0,1, \ldots, 127\}
\end{gathered}
$$

where $\cos \left(\theta\left(t, \mathbf{q}_{j,\{I, Q\}}\right)\right)$ and $\sin \left(\theta\left(t, \mathbf{q}_{j,\{I, Q\}}\right)\right)$ denote the cosine and sine values of phase during $4 T_{\mathrm{b}}$, in which the phase is generated using seven consecutive binary data bits

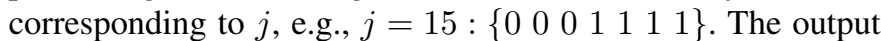
of the receiver is the fourth binary data bit of index $j$ of the maximum correlator output $C_{j,\{I, Q\}}$. The optimum coherent receiver for the F-QPSK-B signal is shown in Fig. 17.

\section{APPENDIX C}

\section{AMP DeCOMPoSition-B ASED Simple MSK-TyPE} COHERENT RECEIVER OF THE F-QPSK-B SIGNAL

When the modulation index of a binary CPM signal is equal to 0.5 , an MSK-type receiver can be used, and the optimum receiver filter is derived using the AMP decomposition of the CPM signal [27]. In this paper, it was shown that the F-QPSK-B signal is a binary CPM signal with a modulation index of 0.5 . 


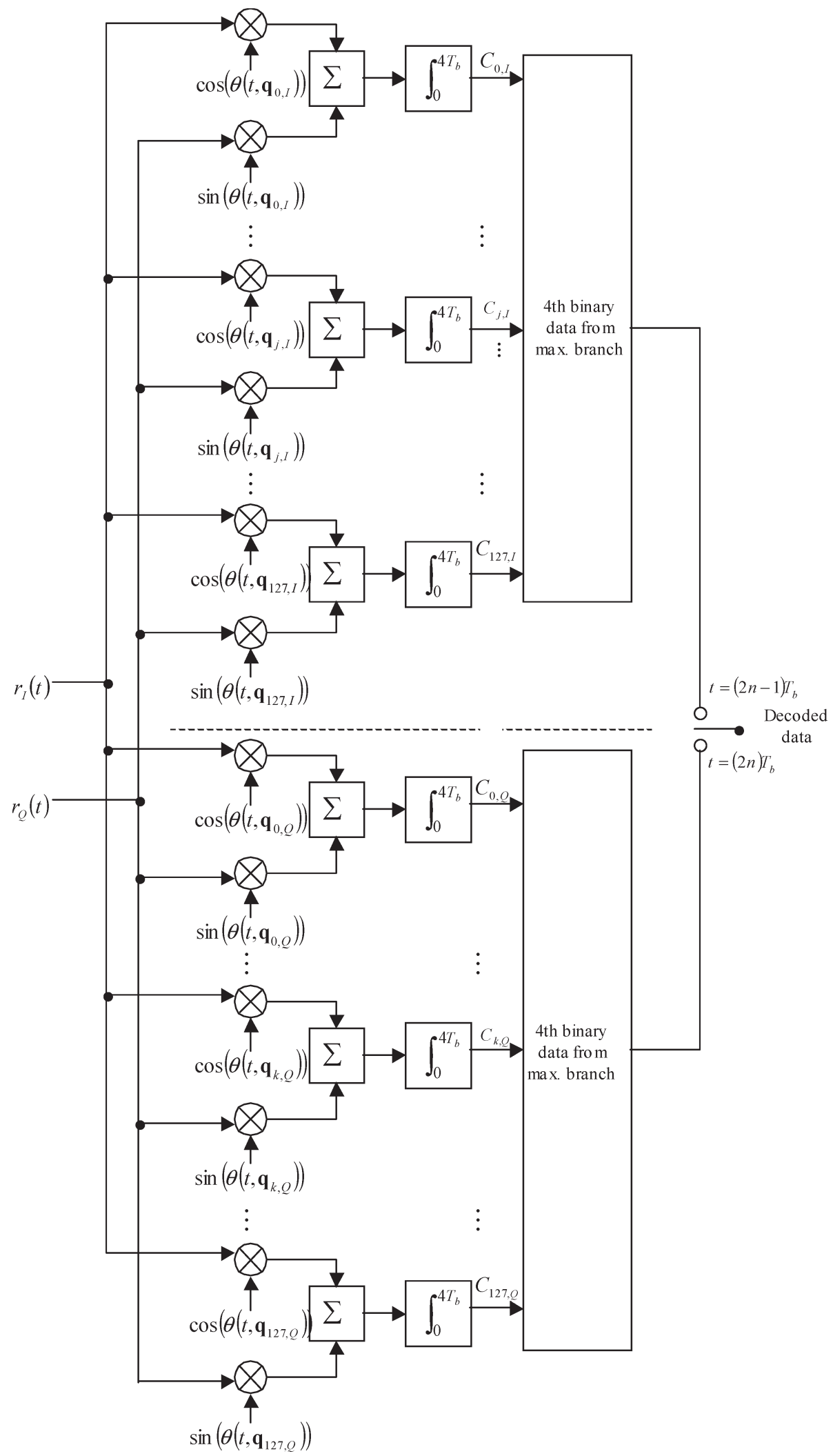

Fig. 17. Optimum coherent receiver of F-QPSK-B signal.

Hence, an MSK-type receiver utilizing the AMP decomposition can be used for the F-QPSK-B signal. In [27], it was shown that the optimum receiver filter for the MSK-type receiver is formed by a Wiener estimator and the most dominant signal component $C_{0}(t)$ of the CPM signal for AMP decomposition. The impulse response of the optimum receiver filter is written as [27]

$$
h(t)=\sum_{k=-N}^{N} w_{k} C_{0}\left(-t+2 k T_{\mathrm{b}}\right)
$$


where $w_{k}$ denotes the coefficient of the Wiener estimator. $C_{0}(t)$ for the F-QPSK-B signal is shown in Fig. 13.

For the received F-QPSK-B signal $z(t), C_{0}(t)$ filter output is written as

$$
r_{0, k}=\int_{-\infty}^{\infty} z(t) C_{0}\left(t-k T_{\mathrm{b}}\right) d t .
$$

Using (C.1) and (C.2), the receiver filter output is written as

$$
y_{n}=\sum_{k=-N}^{N} w_{k} r_{0, n-2 k} .
$$

The optimum coefficients for the Wiener estimator, i.e., $\left\{w_{k} ;-N \leq k \leq N\right\}$, are calculated using the orthogonality conditions for the $I$ channel and the $Q$ channel, as shown in (C.4) and (C.5) [27], [32].

$$
\operatorname{Re}\left\{E\left[\left(a_{0,2 n}-y_{2 n}\right) r_{0,2 n-2 i}\right]\right\}=0, \quad-N \leq i \leq N
$$

$\operatorname{Im}\left\{E\left[\left(a_{0,2 n+1}-y_{2 n+1}\right) r_{0,2 n+1-2 i}\right]\right\}=0, \quad-N \leq i \leq N$.

When the input data is equal to $\alpha_{k}, a_{0, n}$ is defined by [27]

$$
a_{0, n}=(\exp (j \pi h))^{\sum_{k=0}^{n} \alpha_{k}} .
$$

\section{REFERENCES}

[1] T. Cho et al., "A single-chip CMOS direct-conversion transceiver for $900 \mathrm{MHz}$ spread-spectrum digital cordless phones," in Proc. IEEE Int. Solid-State Circuits Conf., 1999, pp. 228-229.

[2] A. Ajjikuttira et al., "A fully-integrated CMOS RFIC for bluetooth applications," in Proc. IEEE Int. Solid-State Circuits Conf., 2001, pp. 198-199.

[3] F. Eynde, J. Craninckx, and P. Goetschalckx, "A fully-integrated zero-IF DECT transceiver," in Proc. IEEE Int. Solid-State Circuits Conf., 2000, pp. 138-139.

[4] P. Orsatti, F. Piazza, and Q. Huang, "A 20-mA-receive, 55-mA-transmit, single-chip GSM transceiver in 0.25- $\mu \mathrm{m}$ CMOS," IEEE J. Solid-State Circuits, vol. 34, no. 12, pp. 1869-1880, Dec. 1999.

[5] B. Razavi, RF Microelectronics. Englewood Cliffs, NJ: Prentice-Hall, 1998.

[6] D. M. Klymyshyn, S. Kumar, and A. Mohammadi, "Direct GMSK modulation with a phase-locked power oscillator," IEEE Trans. Veh. Technol., vol. 48, no. 5, pp. 1616-1625, Sep. 1999.

[7] H. Ishikuro et al., "A single-chip CMOS bluetooth transceiver with 1.5 MHz IF and direct modulation transmitter," in Proc. IEEE Int. Solid-State Circuits Conf., 2003, pp. 94-95.

[8] S. Heinen, S. Beyer, and J. Fenk, "A $3.0 \mathrm{~V} 2 \mathrm{GHz}$ transmitter IC for digital radio communication with integrated VCOs," in Proc. IEEE Int. Solid-State Circuits Conf., 1995, pp. 146-147.

[9] S. Heinen et al., "A $2.7 \mathrm{~V} 2.5 \mathrm{GHz}$ bipolar chipset for digital wireless communication," in Proc. IEEE Int. Solid-State Circuits Conf., 1997, pp. 306-307.

[10] K. Feher et al., U.S. patents $6757334 ; 6665348 ; 6470055 ; 6198777$; 6445749; $6928101 ; \quad 5784402 ; \quad 5491457 ; \quad 4720839 ; \quad 4644565$; $4567602 ; 4350879 ; 4339724$ and 3954926; and pending U.S. patent applications: U.S. S/N 10/205,478; 10/831,562; 10/831,724; 11/023,254; $11 / 023,279 ; 11 / 102,896 ; 11 / 105,295 ; 11 / 107,516 ; 11 / 197,610 ; 11 / 197$, 670; 11/197,609; 09/370,360; 09/370,361; 09/370,362; 09/385,693; $09 / 732,953$.

[11] K. Feher, Wireless Digital Communications. Englewood Cliffs, NJ: Prentice-Hall, 1995
[12] T. Le-Ngoc, K. Feher, and H. Pham Van, "New modulation techniques for low-cost power and bandwidth efficient satellite earth stations," IEEE Trans. Commun., vol. COM-30, no. 1, pp. 275-283, Jan. 1982.

[13] S. Kato and K. Feher, "XPSK: A new cross-correlated phase-shift keying modulation technique," IEEE Trans. Commun., vol. COM-31, no. 5, pp. 701-707, May 1983 .

[14] G. L. Do, "A robust and ultra bandwidth efficient transceiver for interference environment," Ph.D. dissertation, Univ. California-Davis, 1998.

[15] K. Feher, "Spectrally efficiency of commercial wireless and telemetry systems is doubled with IRIG 106-00 standardized FQPSK and is quadrupled with FQAM," in Proc. ITC, 2000, pp. 225-237.

[16] M. K. Simon, Bandwidth-Efficient Digital Modulation With Application to Deep-Space Communications. New York: Wiley, 2003.

[17] Telemetry Standards, Telemetry Group, Range Commanders Council, U.S. Army, White Sands, NM, IRIG Standard 106-00, Jan. 2000.

[18] M. K. Simon and T.-Y. Yan, "Performance evaluation and interpretation of unfiltered feher-patented quadrature phase shift keying (FQPSK)," JPL TMO Progress Rep. No. 42-137, May 1999.

[19] J. G. Proakis, Digital Communications, 3rd ed. New York: McGrawHill, 1995.

[20] J. B. Anderson, T. Aulin, and C. E. Sundberg, Digital Phase Modulation. New York: Plenum, 1986.

[21] H. C. Park, "Non-Coherent detection and fm-vco modulation techniques for quasi-constant amplitude QPSK signals," Ph.D. dissertation, Korea Advanced Inst. Sci. Technol. (KAIST), Daejeon, 2003.

[22] R. R. Anderson and J. Salz, "Spectra of digital FM," Bell Syst. Tech. J., vol. 44, no. 6, pp. 1165-1189, Jul./Aug. 1965.

[23] J. M. Wozencraft and I. M. Jacobs, Principles of Communication Engineering. New York: Wiley, 1965.

[24] T. Aulin, N. Rydbeck, and C. E. Sundberg, "Continuous phase modulation-Part II: Partial response signaling," IEEE Trans. Commun., vol. COM-29, no. 3, pp. 210-225, Mar. 1981.

[25] T. Aulin, C. E. Sundberg, and A. Svensson, "MSK-type receivers for partial response continuous phase modulation," in Proc. IEEE Int. Conf. Commun., 1982, pp. 6F.3.1-6F.3.6.

[26] P. A. Laurent, "Exact and approximate construction of digital phase modulations by superposition of amplitude modulated pulses (AMP)," IEEE Trans. Commun., vol. COM-34, no. 2, pp. 150-160, Feb. 1986.

[27] G. K. Kaleh, "Simple coherent receivers for partial response continuous phase modulation," IEEE J. Sel. Areas Commun., vol. 7, no. 9, pp. 14271436, Dec. 1989.

[28] T. Aulin and C. E. Sundberg, "Continuous phase modulation-Part I: Full response signaling," IEEE Trans. Commun., vol. COM-29, no. 3, pp. 196-209, Mar. 1981.

[29] R. de Buda, "Coherent demodulation of frequency-shift keying with low deviation ratio," IEEE Trans. Commun., vol. COM-20, no. 3, pp. 429-435, Jun. 1972.

[30] T. A. Schonhoff, "Symbol error probabilities for $M$-ary CPFSK: Coherent and noncoherent detection," IEEE Trans. Commun., vol. COM-24, no. 6, pp. 644-652, Jun. 1976.

[31] K. Murota and K. Hirade, "GMSK modulation for digital mobile radio telephony," IEEE Trans. Commun., vol. COM-29, no. 7, pp. 1044-1050, Jul. 1981.

[32] M. H. Hayes, Statistical Digital Signal Processing and Modeling. New York: Wiley, 1996.

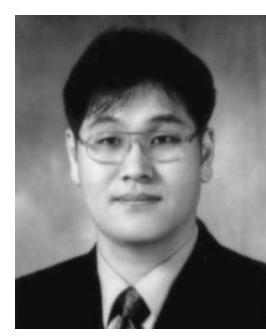

Hyung Chul Park (S'96-M'02) was born in Daegu, Korea, on January 16, 1974. He received the B.S., M.S., and Ph.D. degrees in electrical engineering from the Korea Advanced Institute of Science and Technology (KAIST), Daejeon, in 1996, 1998, and 2003, respectively.

From 2003 to 2005, he was a SoC Design Engineer with Hynix Semiconductor, Seoul, Korea. In 2005, he joined the faculty of the Department of Information, Communication, and Computer Engineering, Hanbat National University, Daejon, where he is currently an Assistant Professor. His current research interests include wireless modulation/demodulation algorithms, system design/implementation, and interface study between RF/IF stages and digital signal processing. 


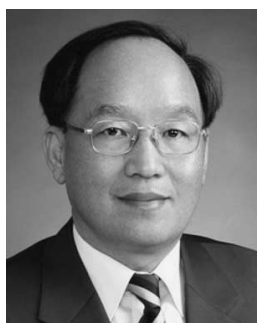

Kwyro Lee (S'79-M'80-SM'89) received the B.S. degree in electronics engineering from Seoul National University, Seoul, Korea, in 1976 and the M.S. and Ph.D. degrees from the University of Minnesota, Minneapolis, in 1981 and 1983, respectively, where he performed pioneering work for characterization and modeling of $\mathrm{AlGaAs} / \mathrm{GaAs}$ heterojunction fieldeffect transistors.

From 1983 to 1986, he was an Engineering General Manager with Gold-Star Semiconductor Inc., Seoul, where he was the Head of the development of the first polysilicon CMOS products in Korea. In 1987, he joined the Korea Advanced Institute of Science and Technology (KAIST), Daejeon, Korea, as an Assistant Professor with the Department of Electrical Engineering. $\mathrm{He}$ is currently a Professor with KAIST. From 1998 to 2000, he served as the KAIST Dean of Research Affairs and the Dean of Institute Development and Cooperation. Since 1997, he has been the Director of Micro Information and Communication Remote Object-oriented Systems (MICROS) Research Center: an Engineering Center of Excellence supported by Korea Science and Engineering Foundation. In March 2005, he joined LG Electronics Institute of Technology, Seoul, as Executive Vice President. He has authored or coauthored over 150 publications in major international journals and conferences. $\mathrm{He}$ authored Semiconductor Device Modeling for VLSI (Prentice-Hall, 1993) and was one of the codevelopers of AIM-SPICE: the world's first SPICE run under Windows.

Dr. Lee is a Life Member of the Korean Institute of Electrical and Communications Engineers. From 1990 to 1996, he served as the Conference Cochair of International Semiconductor Device Research Symposium, Charlottesville, VA. From 1998 to 2000, he served as the Chairman of IEEE Korea Electron Devices Chapter and currently serves as an Elected Member of Administrative Committee of the Electron Devices Society.
Kamilo Feher (F'88) was a Professor of electrical and computer engineering with University of California, Davis, from 1986 to 2003, and prior to that, he was with the University of Ottawa, Ottawa, ON, Canada; Concordia University, Montreal, QC, Canada; Santa Clara University, Santa Clara, CA; and Stanford University, Stanford, CA. He is a President of Digcom, Inc., El Macero, CA, and has been active from 1974 up to the present in consulting and new technology product developments. He has authored six professional books, more than 300 research papers, and has been awarded more than 14 patents, including U.S. patents $6757334,6665348,6470055,6198777,6445749,6928101$ $5784402,5491457,4720839,4644565,4567602,4350879,4339724$, and 3954926 . His patented inventions have been used and licensed to small, medium size, and to some of the largest cellular communications companies for use and applications in 3G wireless systems, the international Global Mobile Systems (GSM), and for use in U.S. Department of Defense and NASA telemetry and satellite systems. These standardized spectrally efficient systems are known as Feher's patented QPSK or FQPSK systems. His quadrature crosscorrelated GMSK modulation inventions have been used and licensed in the implementation of numerous GSM and $3 \mathrm{G}$ products. 